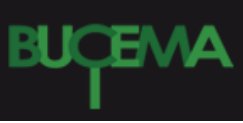

Bulletin du centre d'études médiévales d'Auxerre | BUCEMA

Hors-série $n^{\circ} 10 \mid 2016$

L'origine des sites monastiques : confrontation entre la terminologie des sources textuelles et les données archéologiques

\title{
Locus Novalicii : avant l'abbaye bénédictine de Novalaise
}

\section{Gisella Cantino Wataghin}

\section{QpenEdition \\ Journals}

Édition électronique

URL : https://journals.openedition.org/cem/14478

DOI : $10.4000 /$ cem. 14478

ISSN : 1954-3093

Éditeur

Centre d'études médiévales Saint-Germain d'Auxerre

Référence électronique

Gisella Cantino Wataghin, «Locus Novalicii : avant l'abbaye bénédictine de Novalaise », Bulletin du centre d'études médiévales d'Auxerre | BUCEMA [En ligne], Hors-série nº 10 | 2016, mis en ligne le 09 décembre 2016, consulté le 03 mars 2023. URL : http://journals.openedition.org/cem/14478 ; DOI : https://doi.org/10.4000/cem. 14478

Ce document a été généré automatiquement le 3 mars 2023

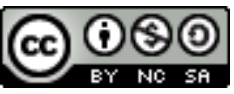

Creative Commons - Attribution - Pas d'Utilisation Commerciale - Partage dans les Mêmes Conditions 4.0 International - CC BY-NC-SA 4.0

https://creativecommons.org/licenses/by-nc-sa/4.0/ 


\title{
Locus Novalicii : avant l'abbaye bénédictine de Novalaise
}

\author{
Gisella Cantino Wataghin
}

1 Ce colloque est l'occasion de revenir sur l'occupation du site de l'abbaye mérovingienne de Novalaise dans les temps qui précèdent sa fondation. Je l'avais déjà évoqué lors d'une communication faite en novembre 2013 à la table ronde internationale de $V$ ienne ${ }^{1}$, restée inédite, après en avoir écrit brièvement le récit dans le passé ; il n'est sans doute pas inutile de reprendre la question aujourd'hui dans le cadre spécifique d'une discussion sur les origines des sites monastiques.

2 L'abbaye de Novalaise est assez connue pour qu'il soit superflu de reprendre dans les détails ses vicissitudes et son rôle, souvent central, dans les relations entre les deux versants des Alpes (fig. 1). 
Fig. 1 - Novalaise, vue aérienne de l'abbaye

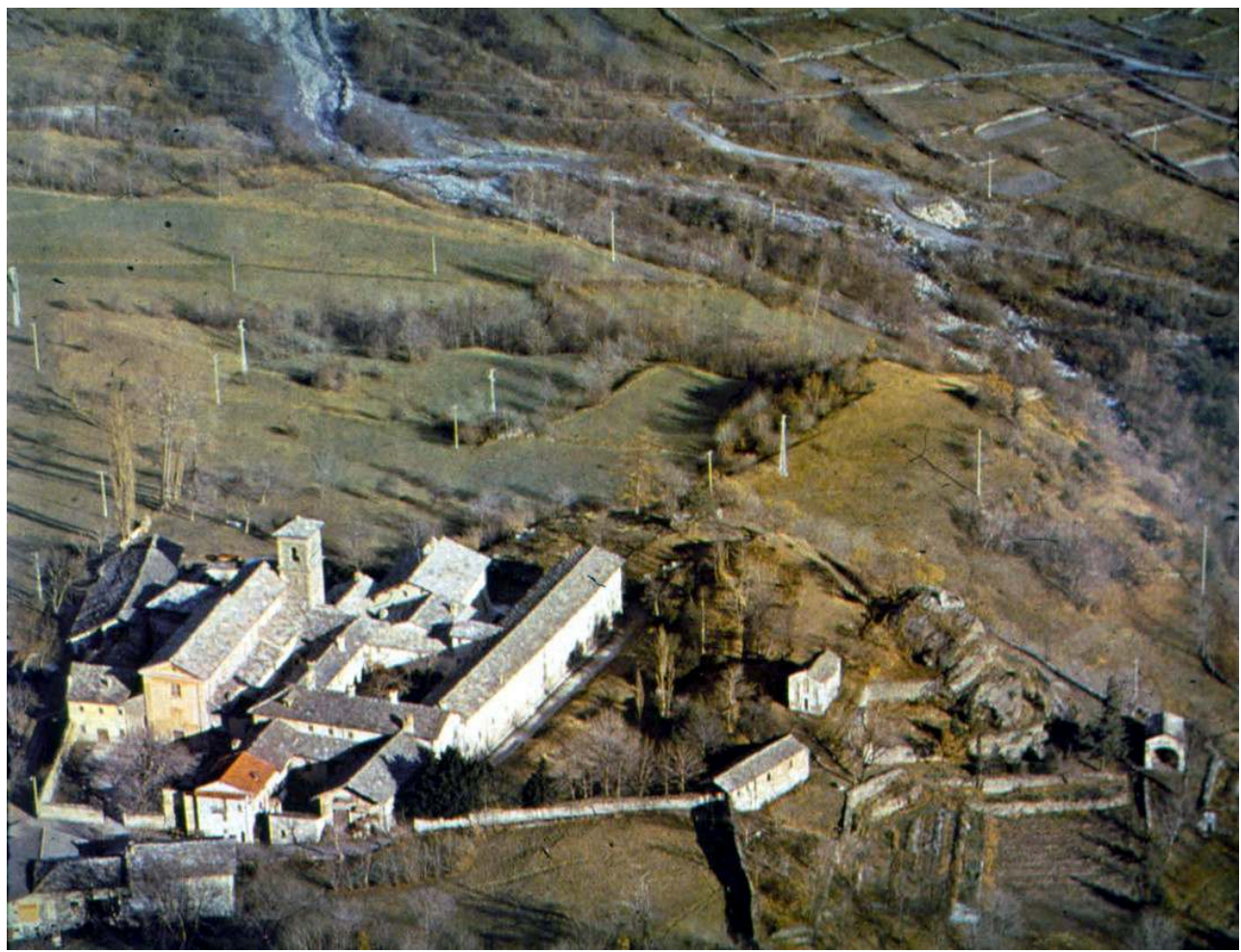

3 Il suffit de rappeler quelques données essentielles. Le monastère est situé au cœur des Alpes, du côté occidental du massif, dans la courte vallée du Cenischia, affluent de la Dora Riparia, au pied du col du Mont-Cenis, à une dizaine de kilomètres au nord-ouest de Suse (fig. 2). 
Fig. 2 - Les Alpes occidentales, vues du satellite, avec l'emplacement de l'abbaye de Novalaise

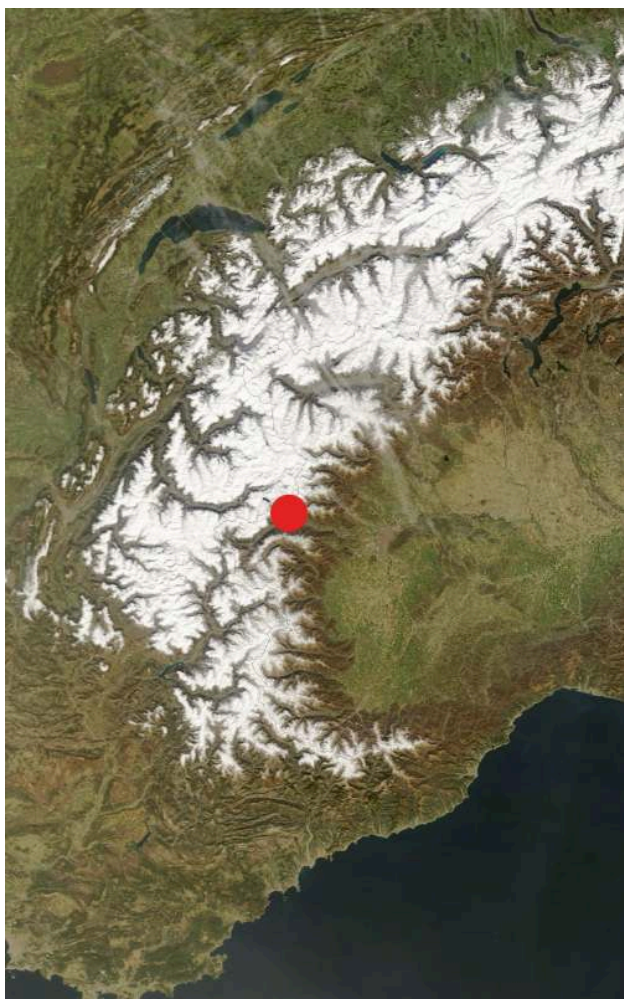

4 La ville, de son côté, donne son nom à la vallée principale, qui aboutit au col du Montgenèvre, et qui, avec le Val Cenischia, assure les liaisons entre l'Italie et la France du Midi, du Centre et du Nord.

5 L'abbaye fut fondée en 726 par Abbon, aristocrate de souche gallo-romaine et haut fonctionnaire du royaume franc, et connut un essor remarquable dans les deux siècles suivants ; abandonnée par la communauté au cours des décennies centrales du $\mathrm{x}^{\mathrm{e}}$ siècle, son renouvellement à la fin de ce siècle comme prieuré dépendant de Breme, dans la Lomellina, où la communauté s'était installée, fut suivi par des hauts et des bas qui parfois en dénaturèrent la fonction, sans pourtant en entamer irrémédiablement les structures $^{2}$. Des interventions du XIXe siècle mises à part ${ }^{3}$, l'état actuel de l'église et du cloître est le résultat d'une reconstruction $d u x_{\text {IIII }}{ }^{e}$ siècle, qui a conservé les dispositions architecturales et une partie non négligeable des élévations du monastère roman; les restes de la phase préromane ont été mis au jour par les fouilles menées à partir de 1978, dans le cadre de la restauration de la plupart des bâtiments ${ }^{4}$.

Dès les années 1960 du siècle passé, quand l'abbaye a fait l'objet d'une enquête pionnière par Giovanni Tabacco ${ }^{5}$, la critique s'est exercée sur le cadre politique de sa fondation, en tant qu'avant-poste de la conquête franque de l'Italie, beaucoup moins sur le contexte de son établissement : en fait, on a tenu pour sûr que la fondation s'était faite à l'écart de la présence humaine, donc dans le cadre du "désert» de l'hagiographie monastique ${ }^{6}$. Si on arrivait parfois à admettre la possibilité d'un modeste habitat romain dans la zone, à caractère sans doute militaire ${ }^{7}$, il était évoqué tout simplement pour expliquer la présence de matériaux romains à l'abbaye, sans approfondir davantage la question.

7 En effet, des matériaux romains étaient connus depuis longtemps à l'abbaye. Déjà au $\mathrm{XVIII}^{\mathrm{e}}$ siècle, Eugenio de Levis signale la présence «Nel giardino dei M. RR. PP. della 
Novalesa » d'une stèle funéraire en marbre (fig. 3-4), dont il connaissait le fragment majeur, l'autre ayant été découvert ensuite, à un moment qu'il est difficile de préciser, mais en tout cas avant le milieu du siècle passé, quand la stèle était murée dans la paroi nord du cloître ${ }^{8}$, tout comme une autre stèle funéraire cintrée 9 .

Fig. 3 - E. DE LEVIS, Raccolta di diverse antiche iscrizioni...tav. IX : stèle romaine

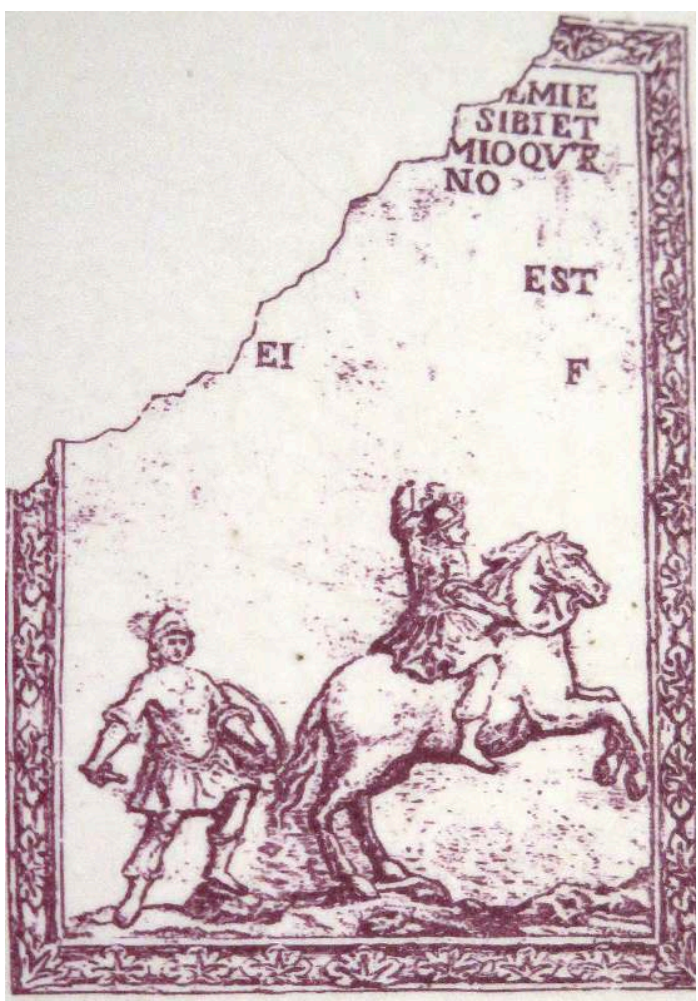

Fig. 4 - Novalaise, Musée Archéologique de l'abbaye : détail d'une stèle funéraire romaine (|ler-\|le siècle)

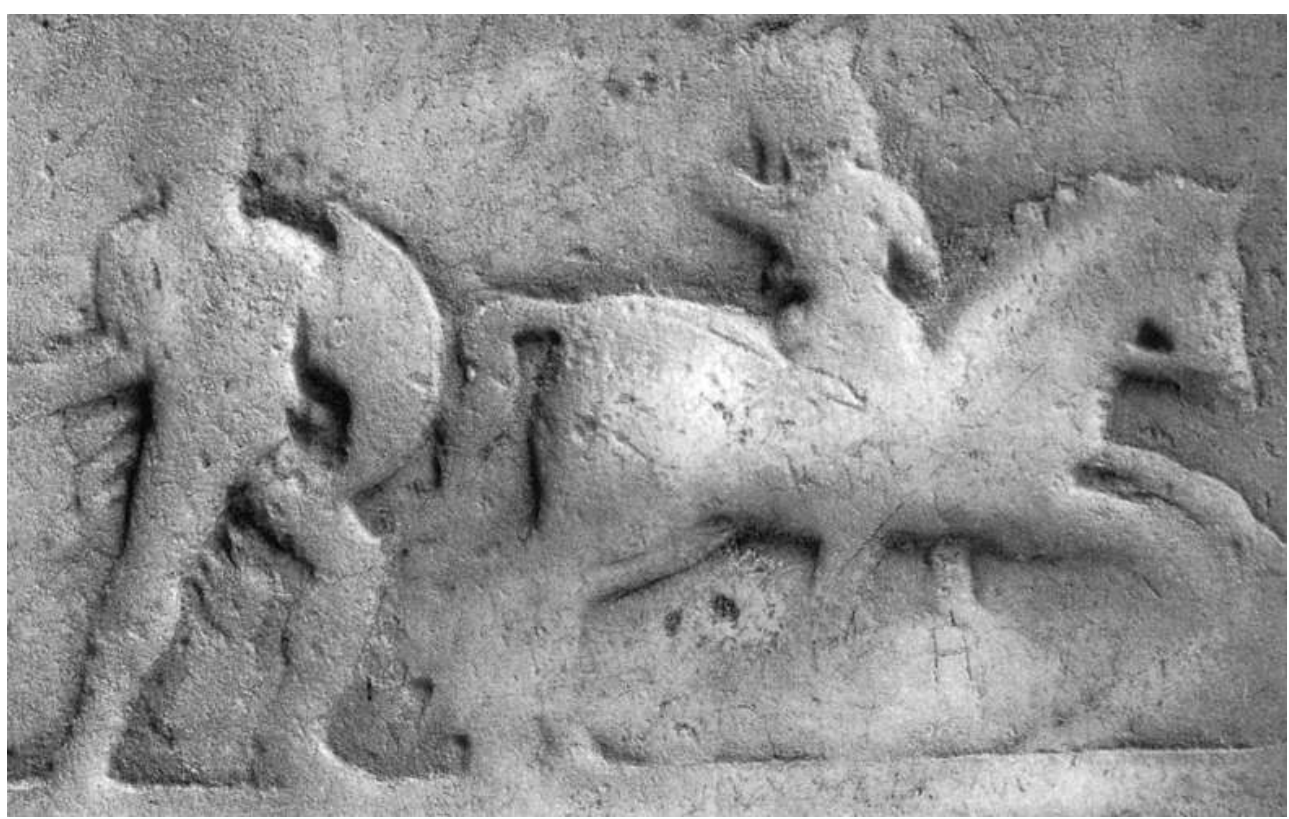


D'autres fragments d'inscriptions, à caractère sans doute funéraire, mais trop menus pour en permettre une lecture satisfaisante, sont également le fruit de trouvailles anciennes ou fortuites, ou bien ont été récupérés au cours des travaux archéologiques récents, toujours en remploi dans les maçonneries ou dans le sol de l'église du $\mathrm{XVIII}^{\mathrm{e}}$ siècle ou bien dans des niveaux de remblais. Il s'agit encore une fois de pièces datables des $\mathrm{I}^{\mathrm{er}}-\mathrm{II}^{\mathrm{e}}$ siècles ${ }^{10}$. Quant aux trouvailles anciennes, aucune information est disponible sur les conditions de leur découverte et les contextes de provenance, ce qui est assez normal pour les XVIII ${ }^{\mathrm{e}}-\mathrm{XIX}{ }^{\mathrm{e}}$ siècles, d'autant plus dans un site comme Novalaise, dont l'histoire récente est assez compliquée, où se succèdent des communautés religieuses différentes et des passages de propriété à des particuliers, qui font des bâtiments d'abord un établissement thermal, ensuite la résidence d'été d'un Internat de Turin. Chacun de ces changements implique des transformations des structures et de leur fonction sur lesquelles nous sommes très mal renseignés ${ }^{11}$. Il en va de même pour les fragments de colonnes, dont l'un est remployé dans l'église abbatiale comme base de l'escalier en colimaçon, qui, dans son angle sud-ouest, donnait accès à la tribune du xvIII ${ }^{\mathrm{e}}$ siècle (fig. 5).

Fig. 5 - Novalaise, Musée Archéologique de l'abbaye : fragments de colonnes, époque romaine

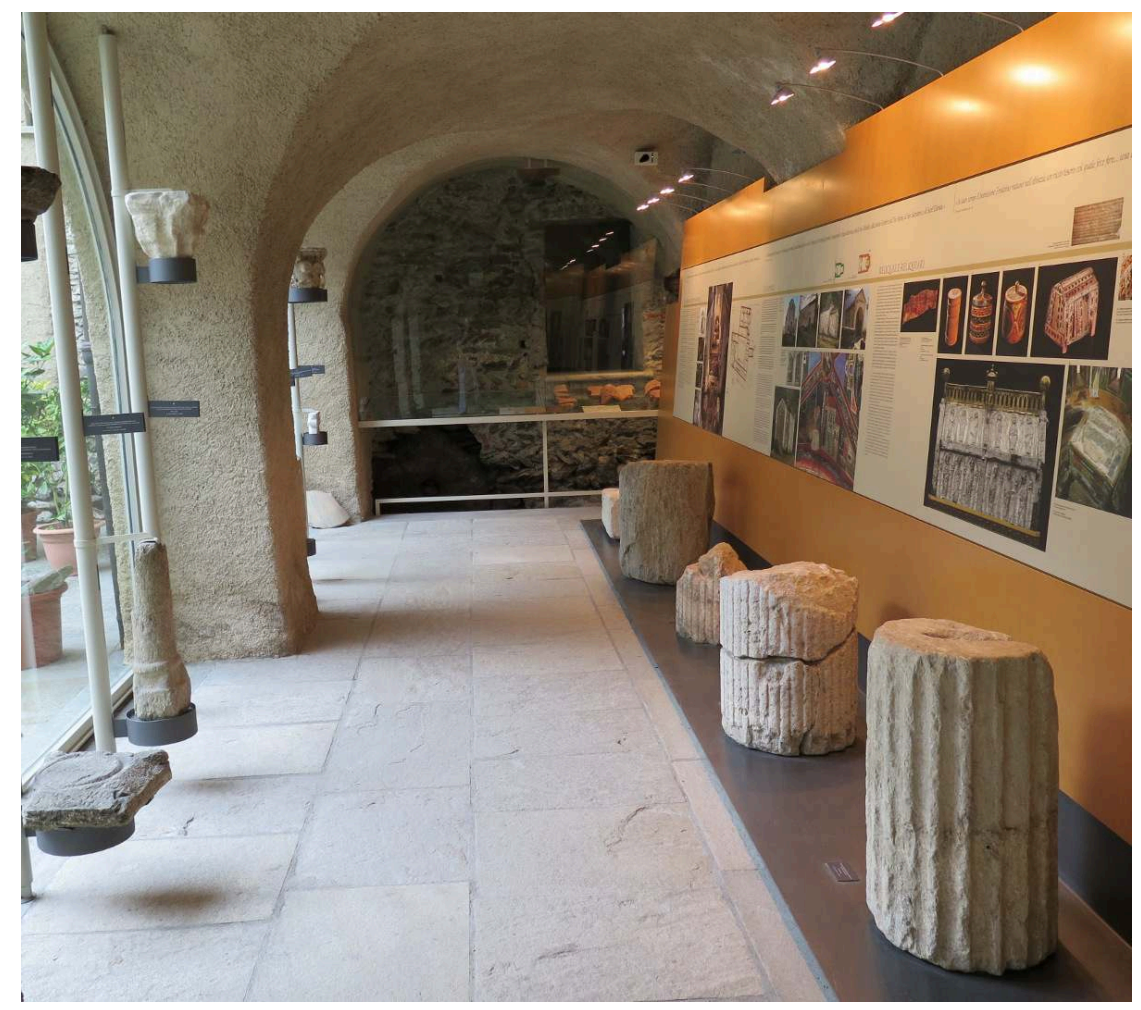

Des deux fragments de chapiteau (fig.6), l'un était également en œuvre dans un contrefort du xviII ${ }^{\mathrm{e}}$ siècle de la paroi orientale de l'aile est du cloître ${ }^{12}$. 
Fig. 6 - Novalaise, Musée Archéologique de l'abbaye : fragments de chapiteaux romains

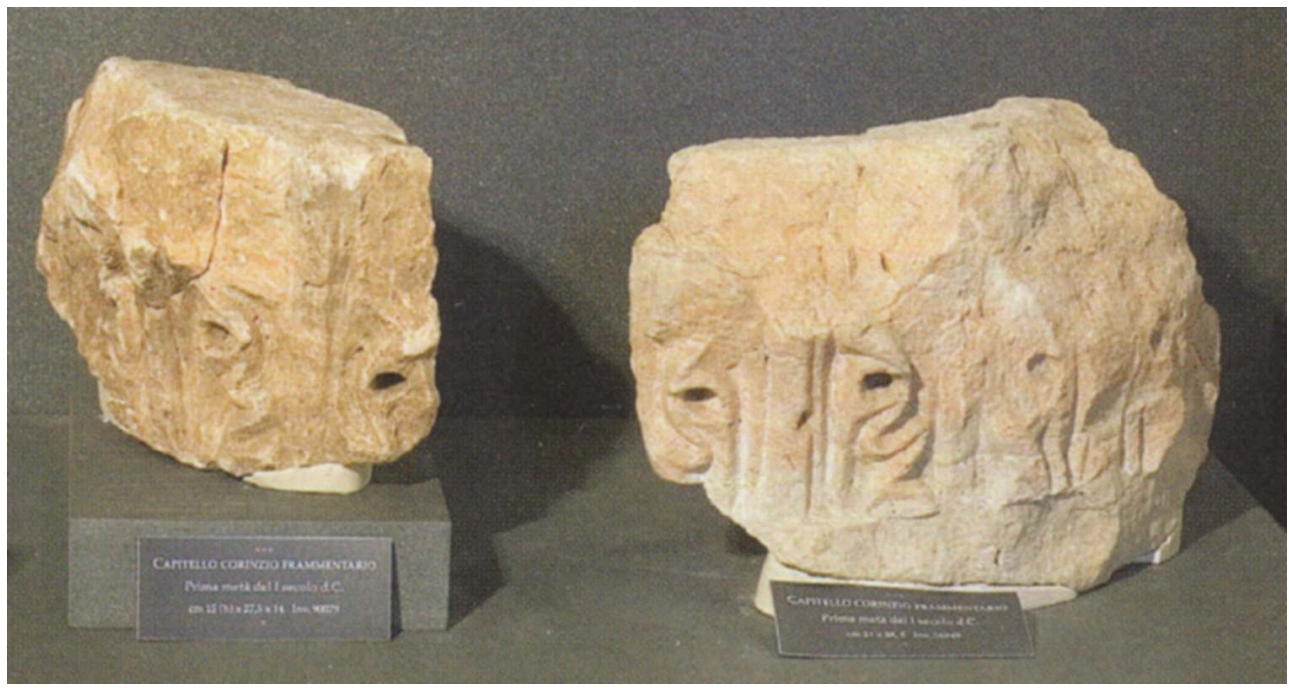

D'autres éléments, également importants et non moins problématiques que les inscriptions, ont été récupérés entre 1973, quand l'abbaye a été achetée par la Province de Turin et qu'une nouvelle communauté bénédictine s'y est installée, et 1978, quand l'archéologie a fait son entrée sur le site, à l'occasion de divers travaux d'aménagement qui ont précédé le démarrage des projets de restauration. Il s'agit notamment d'un fragment de frise décoré d'une panoplie d'armes (fig. 7) et d'un gros bloc architectural (fig. 8-9), décoré sur trois de ses côtés et remployé ensuite comme table de pesage.

Fig. 7 - Novalaise, Musée Archéologique de l'abbaye : frise romaine ('er siècle)

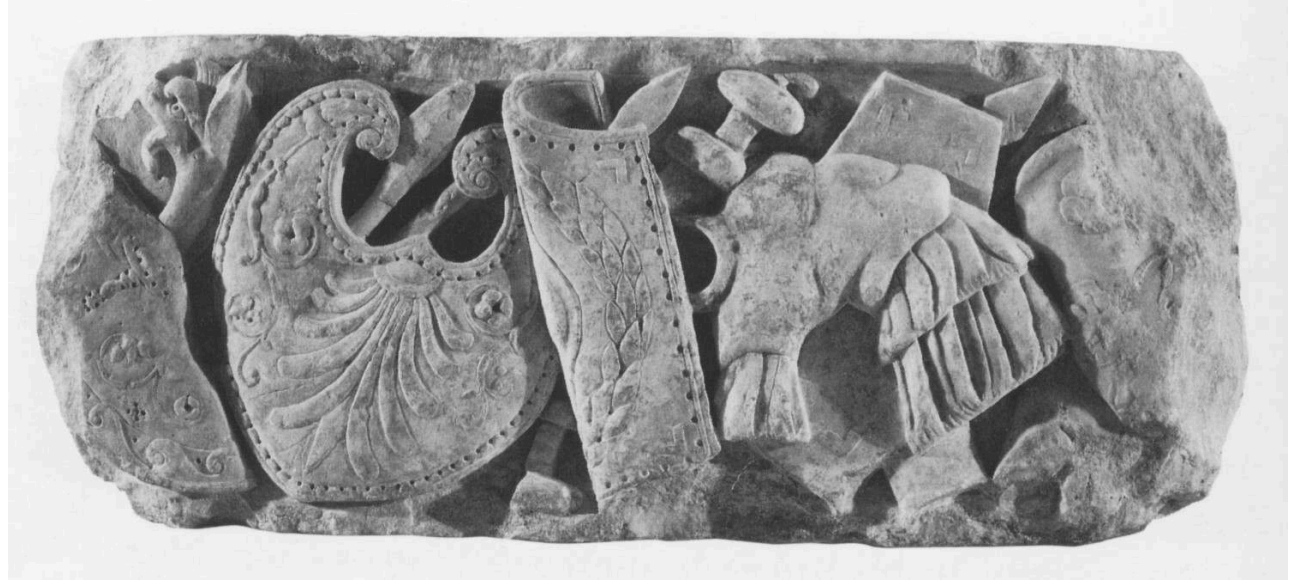


Fig. 8 - Novalaise, Musée Archéologique de l'abbaye : bloc architectural ( ${ }^{\mathrm{er}}$ siècle)

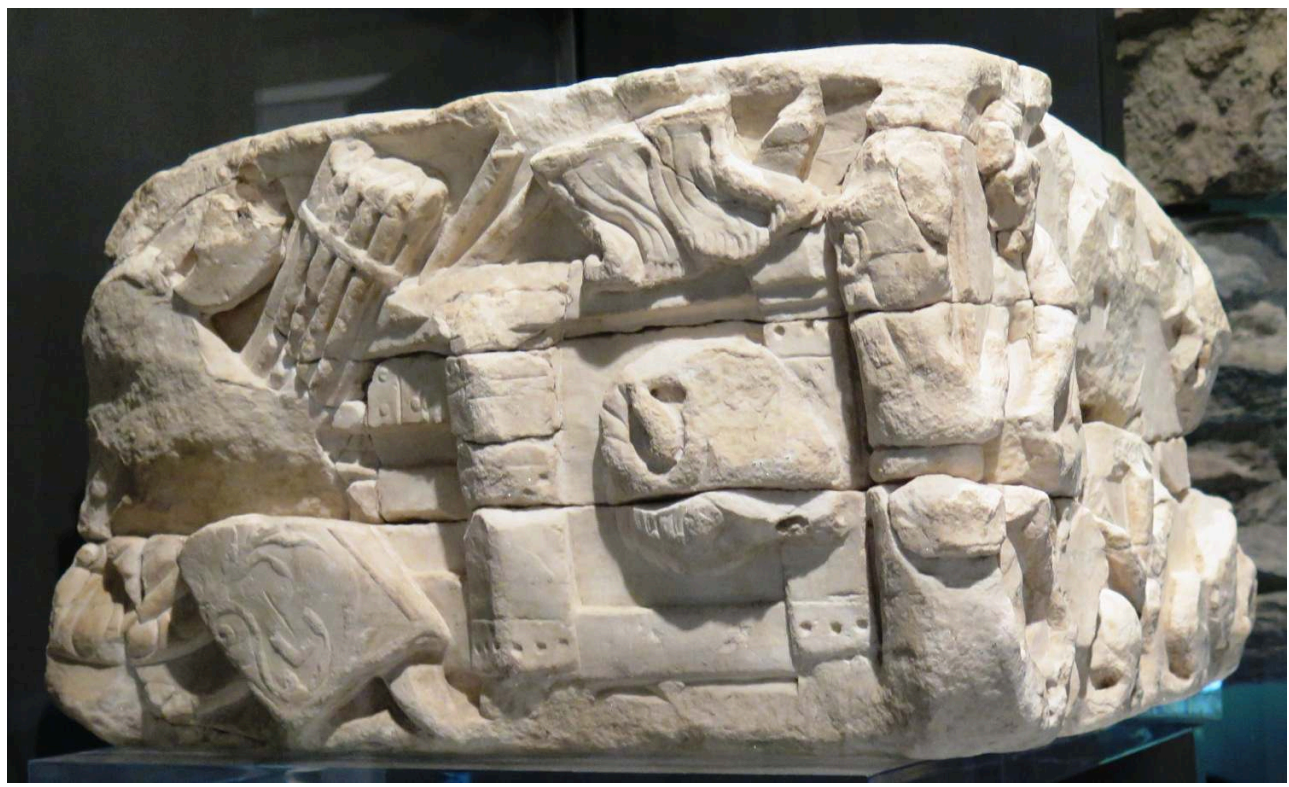

Fig. 9 - Novalaise, Musée Archéologique de l'abbaye : bloc architectural, remployé comme table de pesage

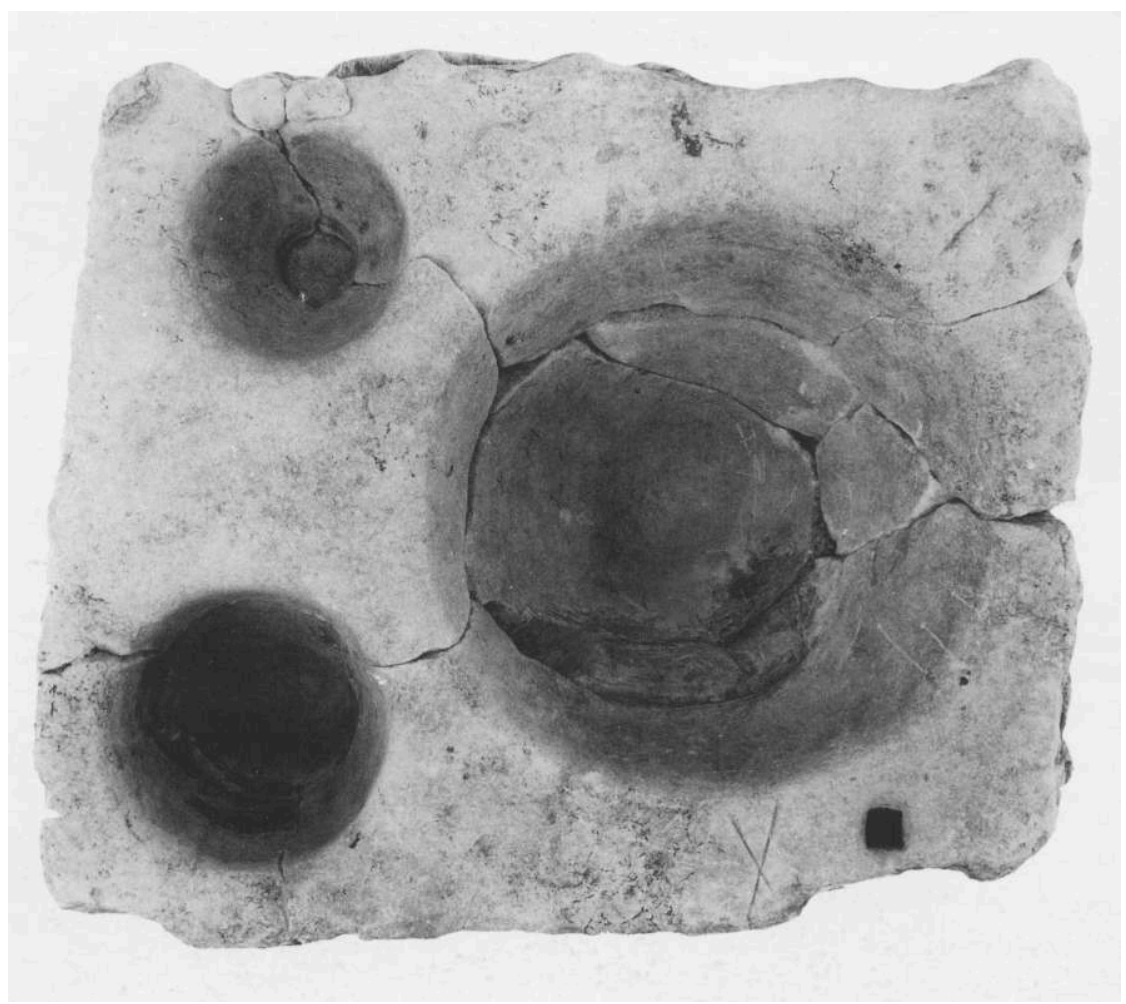

11 L'iconographie de la frise ${ }^{13}$ correspond à celle d'une série de reliefs de Turin, connus dès le $\mathrm{XVIII}^{\mathrm{e}}$ siècle $^{14}$, qui sont datés de l'époque de Claude et ont été attribués récemment à un monument funéraire - plutôt qu'à une statue, dont ils seraient la base -, érigé en l'honneur d'un sénateur provincial, sans doute le père de ce Quintus Glitius Agricola, qui est bien connu dans l'épigraphie locale de l'époque de Trajan ${ }^{15}$. Le fragment de Novalaise peut aussi avoir appartenu à un monument funéraire, tout comme le bloc 
architectural ${ }^{16}$, dont l'ornement est également inspiré de la thématique militaire machine de guerre, armes, panoplie -, conformément à l'idéologie qui s'affirme à l'époque augustéenne ${ }^{17}$. Selon une hypothèse récente, son remploi en tant que table de pesage, suggéré par les trois cavités de dimensions différentes creusées dans sa base et attribué en général au Moyen Âge, dans le cadre de la vie du monastère, pourrait en fait remonter à l'Antiquité - à l'Antiquité tardive, sans doute - les dimensions des cavités étant compatibles avec les unités de volume du système romain ${ }^{18}$. On ne peut que regretter l'absence de données de contexte qui empêche d'aller plus loin dans l'argumentation: en fait, le bloc a été récupéré hors de tout contrôle, dans une puissante couche de remblais à l'extérieur de l'aile sud du cloître, qu'on peut dater du XVII ${ }^{e}$ siècle avancé, quand les Cisterciens ont radicalement modifié l'usage de cette partie du monastère. Il faut quand même souligner que le lieu de la découverte est tout près de l'aile occidentale du cloître, accessible directement de l'extérieur de l'enclos monastique, où les dépendances devaient être installées au Moyen Âge et à la période précédente ${ }^{19}$. Quant à la frise, elle était remployée dans les maçonneries du cloître, notamment dans le montant d'une porte de l'aile orientale, aménagée au XVIII siècle.

Le dossier "romain " s'achève avec deux petites têtes en marbre, elles aussi du ${ }^{\mathrm{er}}$-II ${ }^{\mathrm{e}}$ siècle ${ }^{20}$, quelques fragments de mosaïque (fig. 10) ${ }^{21}$, sans doute romains par leur texture et par la dimension des tesselles, des fragments de briques à poignée et de tuiles de module romain (fig. 11) ${ }^{22}$, des fragments de poterie de l'époque impériale, dont l'importance est inversement proportionnelle à leurs dimensions, tout à fait minuscules ${ }^{23}$.

Fig. 10 - Novalaise, Musée Archéologique de l'abbaye : têtes romaines et fragment de mosaïque

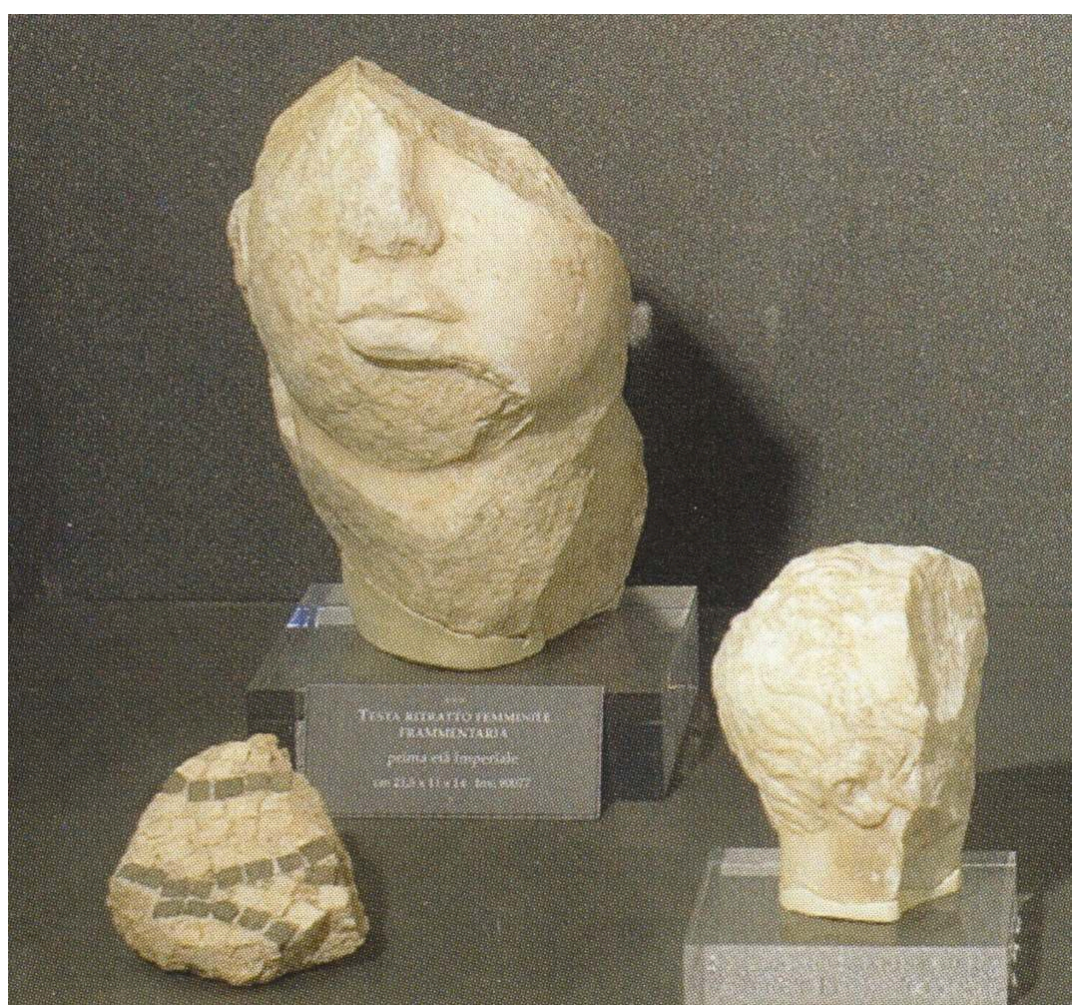


Fig. 11 - Novalaise, Musée Archéologique de l'abbaye : fragments de briques et de tuiles romaines

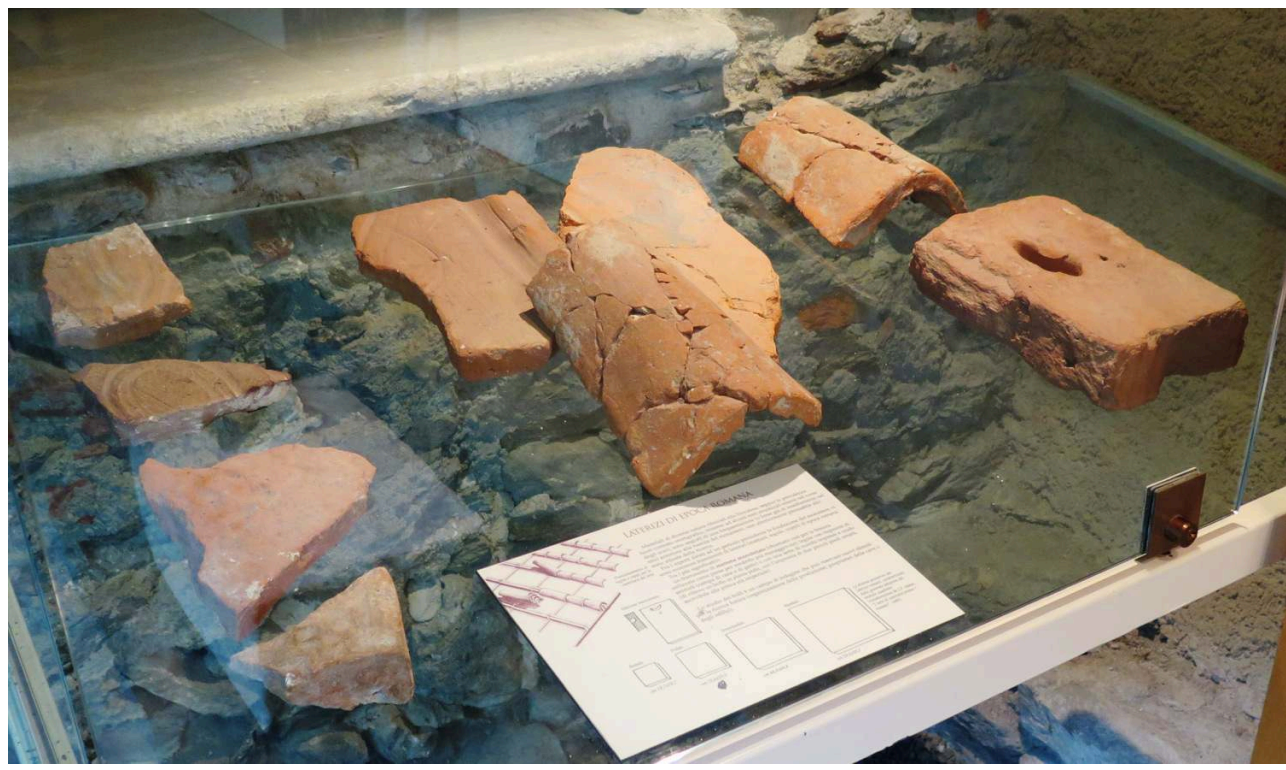

En effet, ces artefacts sont l'indice le plus incontestable de la présence de niveaux d'occupation romains, reconnus par les fouilles seulement dans un petit sondage réalisé à l'extérieur de l'aile sud du cloître (fig. 12).

Fig. 12 - Novalaise : plan archéologique de l'abbaye, avec indication des phases préromanes et romanes (2008)

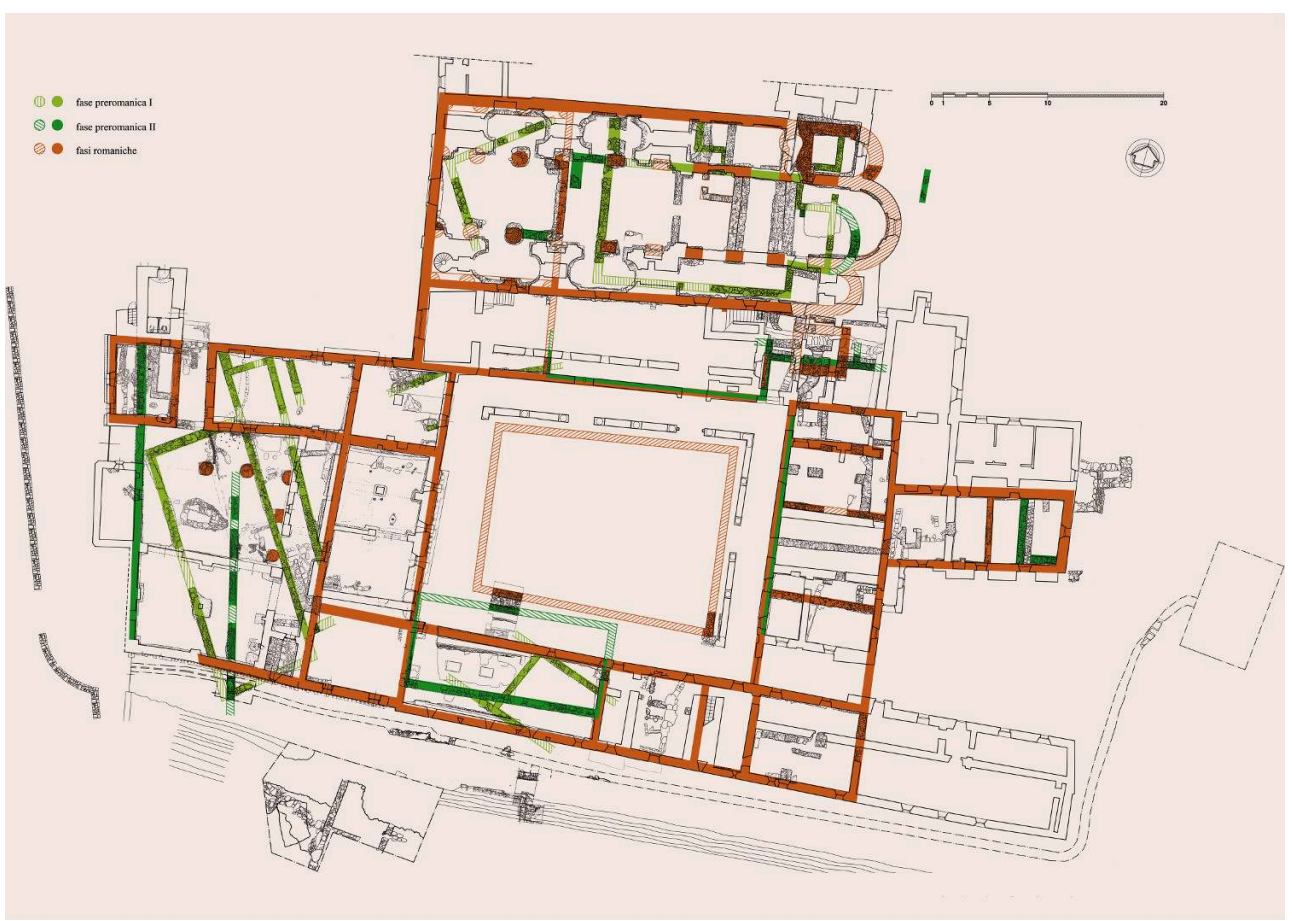

Les limites de ce sondage, qui n'a pas atteint le terrain vierge et n'a pas été suivi par des recherches étendues à l'aire environnante, n'ont pas permis d'interpréter les murs mis au jour ni d'avancer une hypothèse sur la nature de ce contexte ${ }^{24}$.

15 Par conséquent, on ne peut pas exclure que cette occupation ne soit qu'une réponse partielle au problème posé par l'ensemble des matériaux, surtout par ceux, qui, par leur 
nature ou leur dimension, renvoient à des bâtiments importants, qui, à première vue, ne sembleraient pas compatibles avec le site - un site alpestre, on l'a vu, inconnu des sources antiques - et qui semblent justifier l'hypothèse longuement défendue qu'ils aient été récupérés dans la ville toute proche de Suse et remployés dans les maçonneries des bâtiments médiévaux ${ }^{25}$.

Cette possibilité, pourtant, est mise en question par un certain nombre d'arguments, à savoir que les maçonneries médiévales que nous avons pu analyser - appartenant soit à la phase mérovingienne/carolingienne de l'abbaye, soit à sa phase romane - ne montrent aucun élément architectural romain en remploi, les seuls remplois étant des fragments de tuiles, sans doute de l'antiquité tardive ${ }^{26}$, et des fragments de sarcophages, sur lesquels on reviendra sous peu. Il est vrai que la reconstruction du XVIII ${ }^{\mathrm{e}}$ siècle a entraîné des démolitions importantes dans les bâtiments précédents, mais il serait quelque peu surprenant qu'aucune évidence de remplois ne soit reconnaissable dans les maçonneries qui ont survécu. En outre, les fondations du xvIII siècle sont bien plus profondes que celles du Moyen Âge, plus profondes en fait même de la limite de nos fouilles, elles pourraient donc avoir atteint des dépôts antiques.

Des arguments pour avancer dans la réflexion sont offerts par la morphologie de la région et son développement historique.

La vallée du Cenischia (fig. 13-14), dans laquelle se trouve l'abbaye, est secondaire du point de vue géographique par rapport à la vallée de la Dora Riparia, au fond de laquelle le col du Montgenèvre a assuré le passage entre Gaule et Italie dès l'époque protohistorique $^{27}$ - et même avant, si l'on reconnaît un bien-fondé historique au mythe

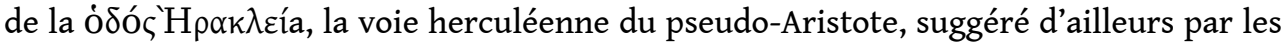
données archéologiques ${ }^{28}$ - et qui est seul attesté par les itinéraires romains qui ne prennent pas en compte le Mont-Cenis ${ }^{29}$ : d'où la thèse traditionnelle selon laquelle celui-ci aurait été "découvert » dans le haut Moyen Âge, à la suite justement de la fondation de l'abbaye de Novalaise ${ }^{30}$. 
Fig. 13 - Le fond du Val Cenischia : au premier plan l'abbaye

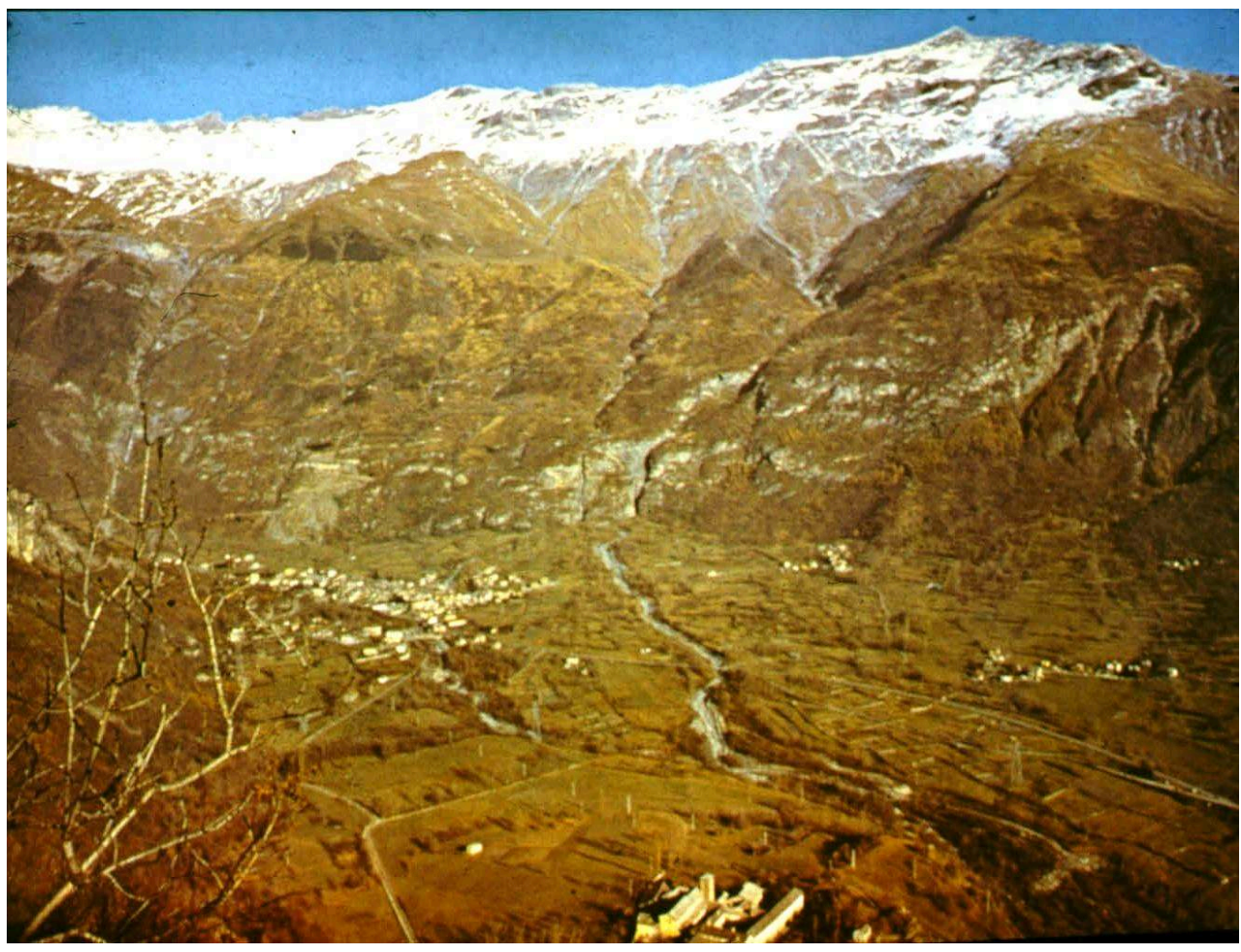

Fig. 14 - Schéma des routes menant de Turin vers la France à travers la vallée de Suse et les cols du Montcenis et du Montgenèvre

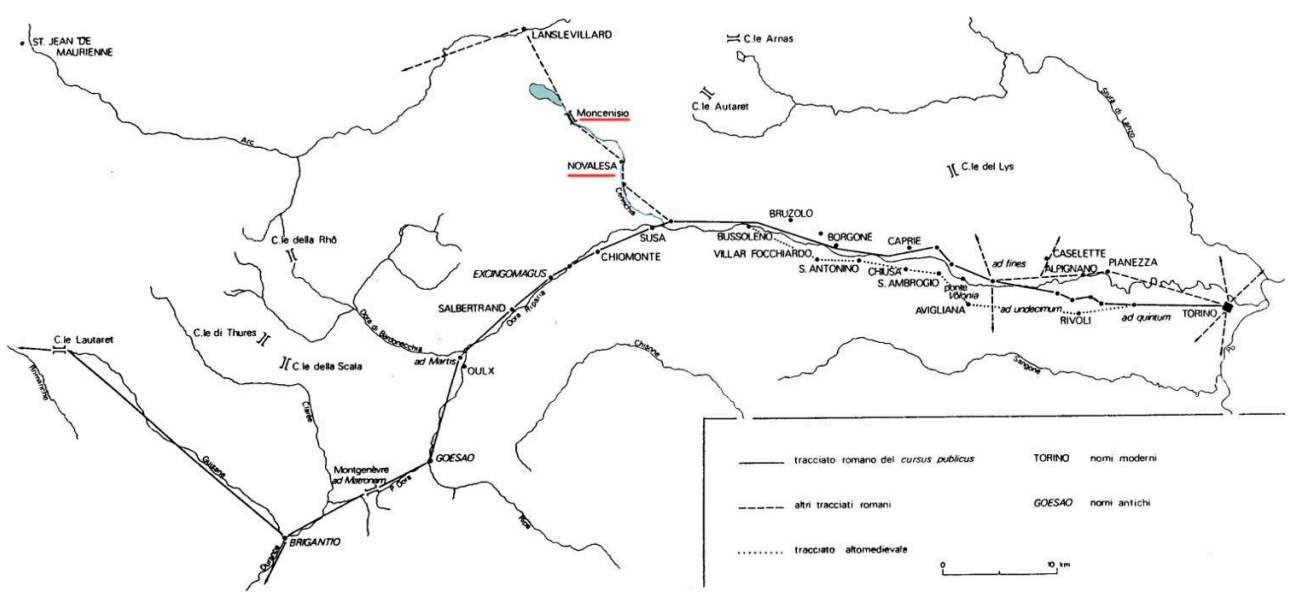

En réalité, on sait bien, d'une part, que les itinéraires ne sont pas exhaustifs du réseau routier romain ${ }^{31}$ et, d'autre part, il est vraisemblable que ce col fut aménagé dans le cadre de la romanisation de la région. Des découvertes archéologiques sur le versant français, qui en sont un signe évident, ont été signalées depuis longtemps ${ }^{32}$. Plus récemment, les inscriptions $d u \mathrm{I}^{\mathrm{er}}$ siècle ap. J.-C., repérées en 1991 du côté français du Mont-Cenis, à une hauteur de plus de $2000 \mathrm{~m}$, sur un rocher portant déjà une scène de chasse datant du ve siècle av. J.-C., ont confirmé la fréquentation non occasionnelle du col, qu'on a supposé en relation avec le contrôle du commerce entre les Gaules et l'Italie $^{33}$. Du côté italien, le passage régulier et organisé est attesté par le fragment de milliaire retrouvé hors contexte à l'abbaye ${ }^{34}$. La fréquentation régulière du Mont-Cenis entre d'ailleurs dans une logique d'exploitation des différents parcours à travers les 
Alpes. Le rapport entre les deux cols, dans la perspective d'une hiérarchie, est un faux problème : ils étaient complémentaires et non pas alternatifs, ce que l'emplacement de la ville de Suse, au confluent des deux vallées de la Doire Riparia et du Cenischia, montre ouvertement ${ }^{35}$.

Faute de pouvoir dater le milliaire, le fragment ne présentant pas d'inscription, on remarquera que si le col du Montgenèvre donne accès à la vallée de la Durance, et donc à la Gaule méridionale et à Lyon et à sa région par le col du Lautaret, pourtant d'accès difficile, le passage du Mont-Cenis est le plus direct vers la Gaule du Centre/Nord, qu'il revêt donc une importance particulière dès le deuxième quart du $\mathrm{I}^{\mathrm{er}}$ siècle, quand l'empereur Claude projette et réalise, entre 43 et 57, la conquête de la Bretagne. Ce n'est pas un hasard si, en 44, la préfecture des Alpes Cottiennes est promue au rang de royaume sous Cottius II, bien connu par ses évergésies à Turin ${ }^{36}$, ni si la ville de Suse connaît un développement monumental important à l'époque claudienne, comme le démontre avec force et arguments une étude récente ${ }^{37}$. Même de courte durée - il devient province sous Néron ${ }^{38}$-, le Cottii regnum reste ancré dans la mémoire: on le retrouve encore au $\mathrm{IV}^{\mathrm{e}}$ siècle sur la Tabula Peutingeriana ${ }^{39}$. Toujours au IV siècle, Ammien évoque l'aménagement des voies des Alpes - au pluriel, il est à remarquer - de la part de Cottius, le premier ou le deuxième de ce nom, ou sans doute les deux ${ }^{40}$. Dans ce cadre, l'établissement d'un point d'appui au pied du col du Mont-Cenis, dont le passage est souvent difficile à cause de l'altitude, du climat et de l'escarpement du versant italien, ne serait que logique : une statio, dotée de bâtiments importants, peutêtre développée en agglomération, comme c'est le cas entre autres de la statio ad Quintum, elle aussi sur la voie des Gaules, près de Turin ${ }^{41}$ ? Ou plutôt une villa, dont la fonction de centre d'exploitation d'un domaine rural se doublerait de fonctions d'accueil liées au passage et dont la proximité à la ville de Suse assurerait une identité, le cas échéant monumentale, de villa suburbaine ${ }^{42}$ ? Ce n'est certes pas par hasard si le toponyme "Novalicii » vient du latin novalis, terre récemment mise en culture ${ }^{43}$, ce qui marque la romanisation de la vallée et donc la mise en valeur, sans doute par les notables, de ses ressources - terres arables, prairies, forêts, pâturages, minières ${ }^{44}$. Le point d'interrogation s'impose, mais aussi la remarque que la difficulté à admettre une telle possibilité dans une vallée alpestre tient beaucoup du stéréotype de la montagne sauvage et inhabitable et en même temps des limites de nos connaissances des formes qui peuvent résulter de son occupation. Il n'est sans doute pas hors de propos d'évoquer les implications de l'inscription de Quartinus / Buss]ulli filius et de sa famille, dont le préfet Albanus ${ }^{45}$, appartenant à un important monument funéraire familial érigé au hameau des Escoyères, situé dans le Queyras, à $1550 \mathrm{~m}$ d'altitude, accessible seulement par une piste de hauteur, pourtant parcourue dans le cadre d'un système routier «secondaire » de liaison des différentes vallées alpestres ${ }^{46}$. Il faut également signaler le mausolée monumental d'Argenton, dans la petite commune des Alpes du Sud de Fugeret, à $1300 \mathrm{~m}$ d'altitude, entre les hautes vallées du Verdon et du Var, à l'écart des voies les plus fréquentées, attribué à un chevalier romain qui y aurait possédé un domaine ${ }^{47}$.

21 Ce qui est certain, c'est que l'occupation du site ne connaît pas d'interruption à l'Antiquité tardive, mais semble se développer, au contraire, sur une échelle étendue non négligeable, comme l'attestent les fragments de tuiles remployés dans des maçonneries romanes, dont il a déjà été question, datés par thermoluminescence du $\mathrm{IV}^{\mathrm{e}}-\mathrm{V}^{\mathrm{e}}$ siècle $^{48}$, et le mobilier céramique et en pierre ollaire ${ }^{49}$. Un contexte funéraire est 
suggéré par des matériaux dispersés, tels un peigne (fig. 15) et un fragment de gaine en os (fig. 16) du VI ${ }^{\mathrm{e}}-\mathrm{VII}^{\mathrm{e}}$ siècle $^{50}$.

Fig. 15 - Novalaise, Musée Archéologique de l'abbaye : peigne en os, $\mathrm{Vl}^{\mathrm{e}}-\mathrm{VII}^{\mathrm{e}}$ siècle

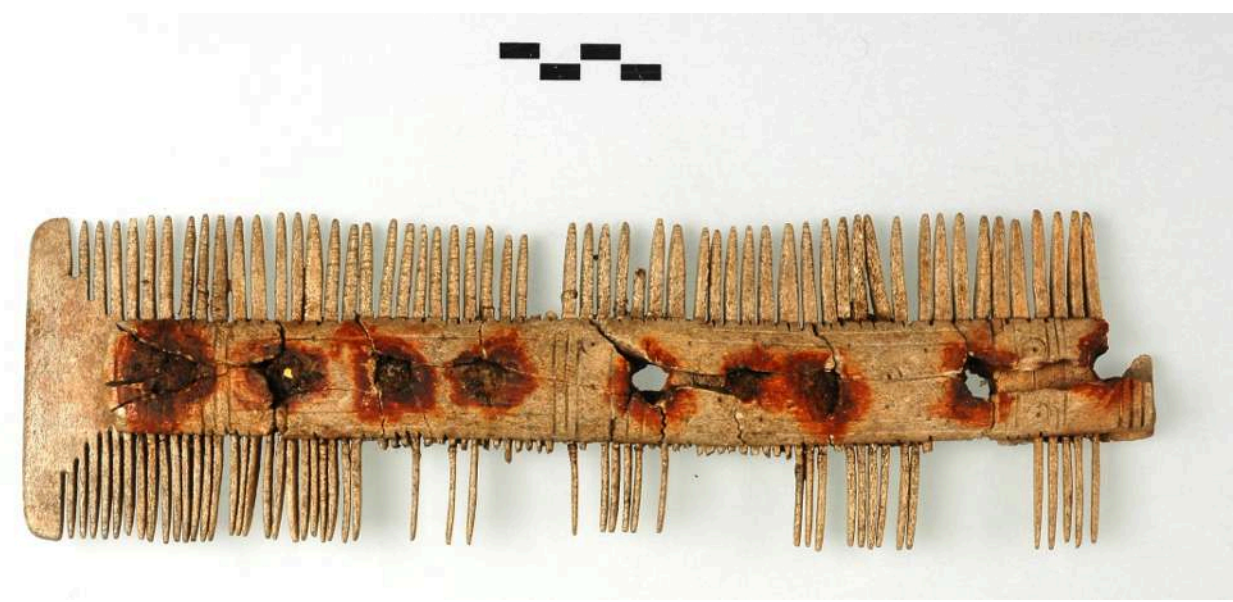

F. Crivello et c. segre montel (dir.), Carlo Magno e le Alpi. Viaggio al centro del Medioevo, Milan, 2006.

Fig. 16 - Novalaise, Musée Archéologique de l'abbaye : élément de gaine de peigne en os, vie$\mathrm{VII}$ siècle

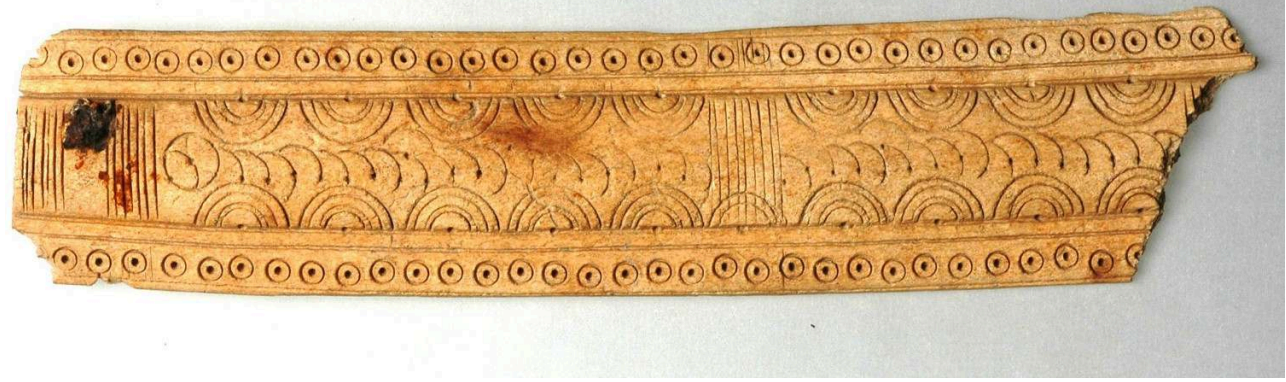

F. Crivello et c. segre montel (dir.), Carlo Magno e le Alpi. Viaggio al centro del Medioevo, Milan, 2006.

Des $\mathrm{VI}^{\mathrm{e}}-\mathrm{VII}{ }^{\mathrm{e}}$ siècles, date aussi le reliquaire en os retrouvé dans la chasse médiévale de Saint-Eldrade ${ }^{51}$ (fig. 17), de production vraisemblablement mérovingienne, comme l'indique l'affinité avec le reliquaire de Brédons ${ }^{52}$, mais malheureusement rien ne permet de préciser depuis quand cet objet est présent à l'abbaye ${ }^{53}$. 
Fig. 17 - Susa, Museo Diocesano di Arte Sacra : reliquaire ( $\mathrm{Vl}^{\mathrm{e}}-\mathrm{VII}{ }^{\mathrm{e}}$ siècle)

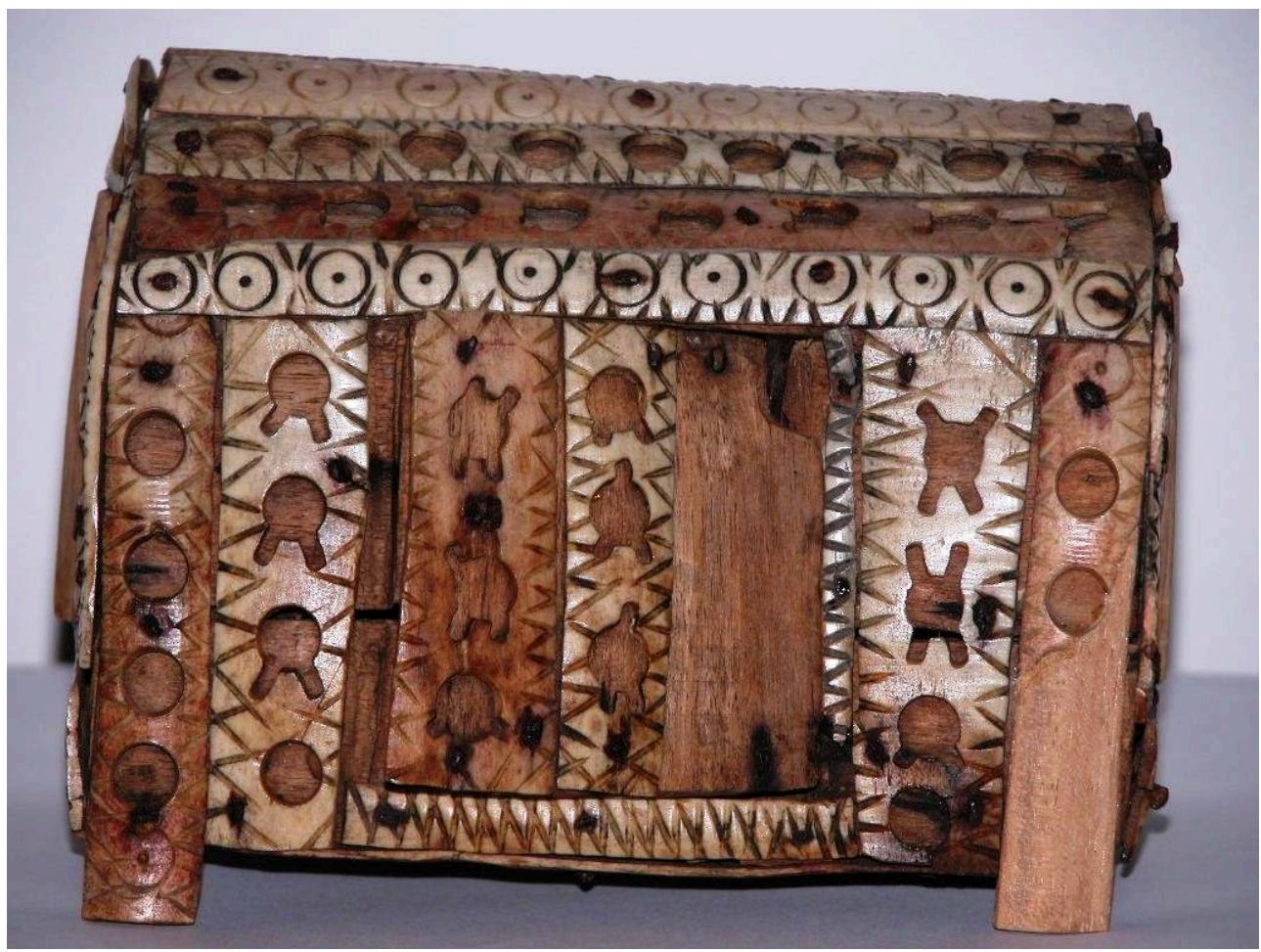

G. SARONI, « Cofanetto reliquiario in osso », in Novalesa. Una storia tra fede e arte, Sant'Ambrogio di Torino, 2000. dans des niveaux de remblai ou remployés dans des maçonneries du xvIII siècle, ont vraisemblablement été transportés d'ailleurs pour servir de sépultures privilégiées au haut Moyen $\hat{A}_{\mathrm{g}} \mathrm{e}^{54}$. Quant à l'inscription métrique fragmentaire, gravée sur un bloc, décorée sur l'autre côté d'un motif d'entrelacs ${ }^{55}$, elle appartient avec toute vraisemblance au haut Moyen Âge et non pas à l'Antiquité tardive, comme on l'a souvent proposés ${ }^{5}$.

De surcroît - et c'est la donnée la plus importante -, des structures préexistantes ont été remployées au moment de l'aménagement des premiers bâtiments monastiques, au début $\mathrm{du}$ VIII ${ }^{\mathrm{e}}$ siècle. Elles ont été mises au jour dans les ailes sud et ouest du cloître roman (cf. fig. 12 et 18), ainsi que dans la cour devant l'église abbatiale ${ }^{57}$. 
Fig. 18 - Cour de l'aile ouest du cloître : restes des édifices antérieurs à la phase carolingienne (Soprintendenza Archeologia Piemonte)

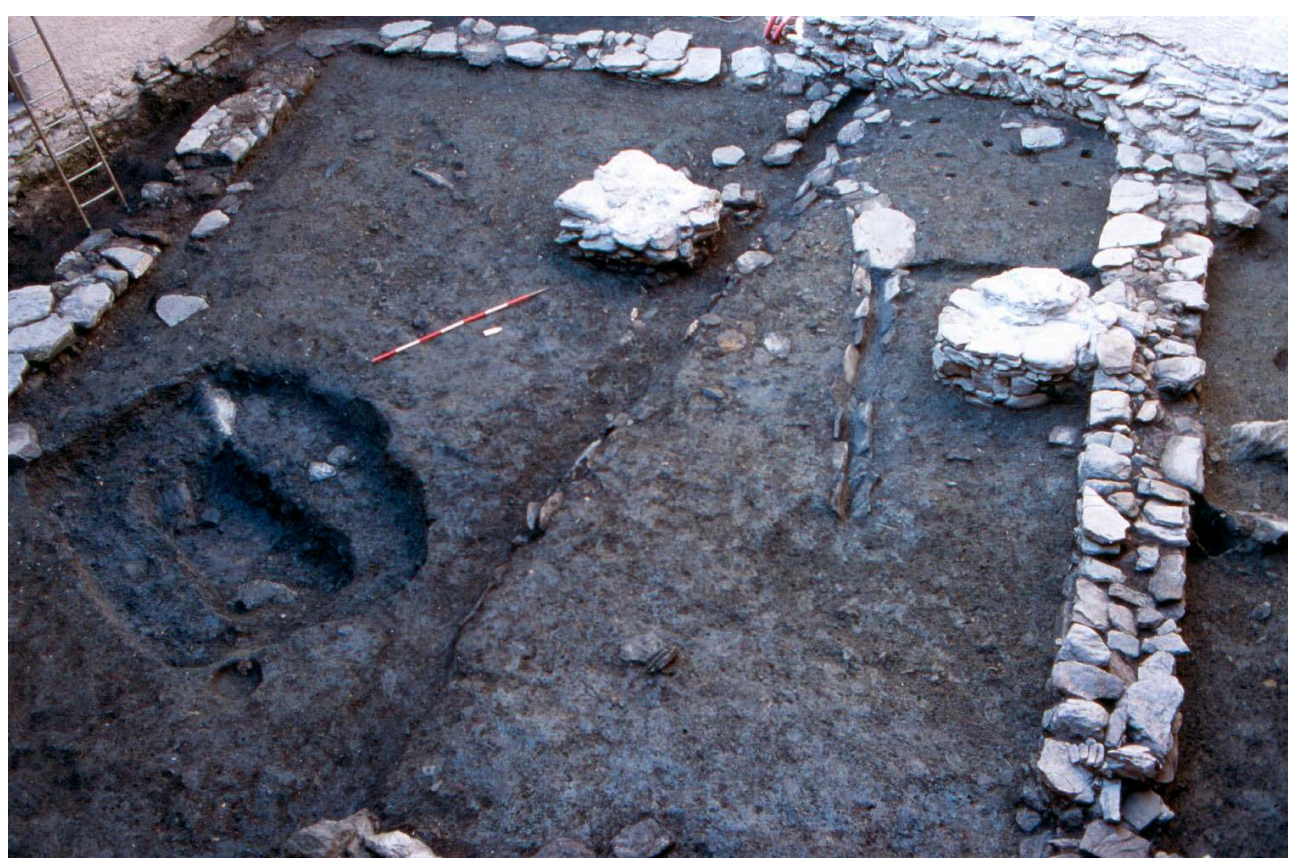

Elles se signalent par leur disposition en biais, par rapport à l'orientation de la première église abbatiale, qui devient ensuite celle des autres édifices du monastère. Bien qu'elles ne soient préservées qu'au niveau de fondation, il est assez évident que se dessine un schéma de bâtiments rectangulaires allongés, sans doute flanqués sur l'un des longs côtés d'un couloir ou portique, ce qui n'est pas sans évoquer une typologie bien connue entre l'Antiquité tardive et le haut Moyen Âge. Un sol en mortier solide sur radier de gros cailloux et de pierres est présent dans l'espace mis au jour dans l'aile sud du cloître, en dessous du réfectoire carolingien.

26 Il serait évidemment important de savoir quels sont l'état et la fonction de ces bâtiments, au moment où les moines les occupent. Concernant le premier point, dans aucun endroit, la fouille n'a montré des traces d'abandon, ce qui suggère une continuité d'occupation entre ces édifices et les structures qui marquent au $\mathrm{IX}^{\mathrm{e}}$ siècle la restructuration du monastère ${ }^{58}$. Sur le second, on se bornera à remarquer la complexité des bâtiments mis au jour et leur disposition, assez cohérente du point de vue de l'orientation, même si elle n'est pas tout à fait régulière et qu'elle ne montre pas un système de distribution évident, ce qui suggère un habitat assez articulé. Sa relation avec l'établissement romain reste à préciser, tout comme la nature et le statut de celuici, et son statut et bien sûr les voies par lesquelles ce domaine parvient aux mains d'Abbon qui y installe une communauté de moines.

Le privilège, indiqué couramment comme acte de fondation du monastère (726), et le testament d'Abbon (739) se réfèrent au site comme locus nunccopans Novalicii. Le mot locus, que Ganshof avait interprété comme synonyme de villa ${ }^{59}$ et, qui, selon l'étude récente de Jean-Michel Carriée ${ }^{6}$, ferait allusion à une forme d'occupation du sol, vraisemblablement agricole, intégrée dans un fundus ou bien assimilable à celui-ci selon les cas, semble avoir ici tout simplement la signification générique de toponyme - dans le sens de " lieu-dit $»^{61}$. Toutefois, Abbon ajoute in rem proprietatis nostrae: il s'agit donc en tout cas d'une unité de son immense patrimoine, situé dans la région de Suse et 
surtout du côté français des Alpes, le long des vallées du Rhône et de la Durance, et en Provence (fig. 19) ${ }^{62}$.

Fig. 19 - Les possessions de l'abbaye d'après le testament d'Abbon

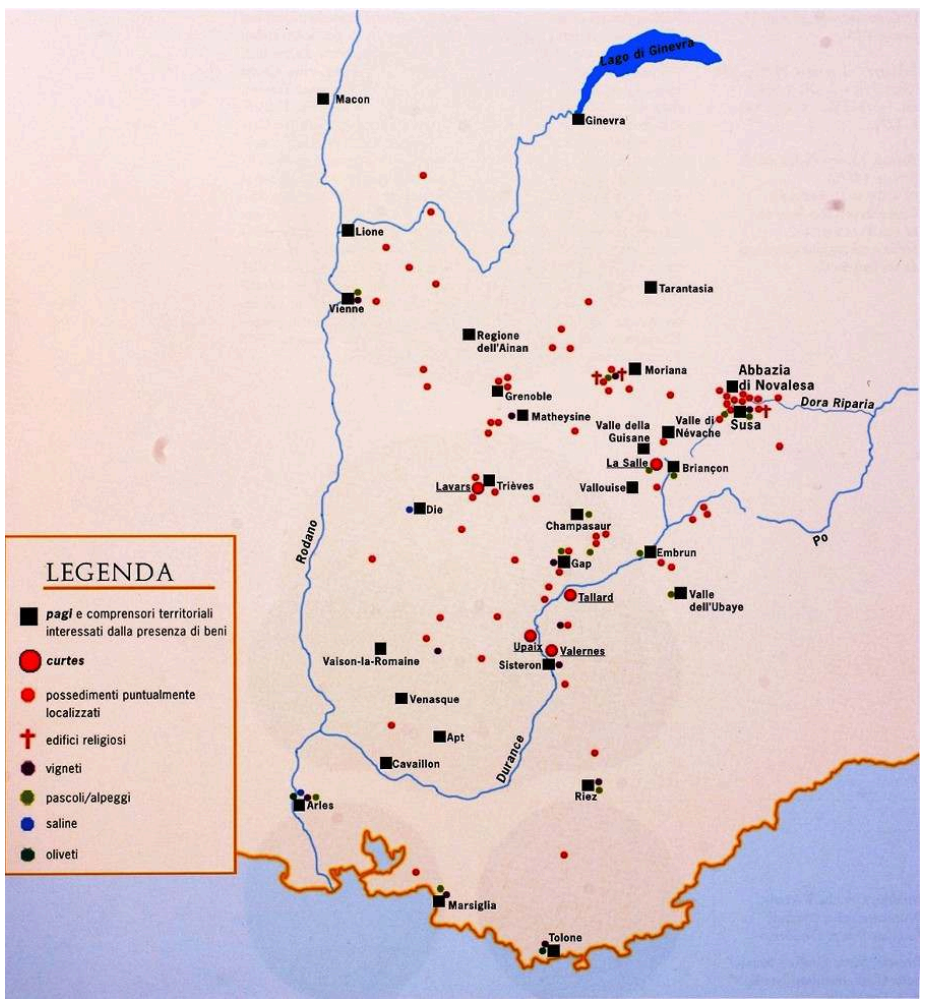

E. Destefanis et s. UGgè, « Possedimenti di Novalesa secondo il testamento di Abbone 739 », in F. Crivello et c. segre montel (dir.), Carlo Magno e le Alpi. Viaggio al centro del Medioevo, Milan, 2006.

Dans cette géographie complexe, le locus Novalicii représente le noyau des propriétés léguées à Abbon par la famille de son père, juste au cœur de la région de Suse et de la Maurienne, dont il est rector "par la grâce de Dieu " ${ }^{63}$ : un " lieu » tout près de Suse, sans doute le centre de gestion de son patrimoine, un rôle dont l'abbaye ne serait que l'héritière ${ }^{64}$.

Sa localisation dans le Val Cenischia et son rapport privilégié avec la route soi-disant secondaire du Mont-Cenis ne doit pas nous fourvoyer, la logique des communications dans l'Antiquité tardive et le haut Moyen Âge n'étant pas celle des autoroutes actuelles. D'une part, d'autres possessions d'Abbon sont également présentes dans la vallée de la Dora Riparia, donnant accès direct à la vallée de la Durance et donc à la Provence. D'autre part, le patrimoine s'étend jusqu'à Vienne, à Lyon et au Mâconnais, avec des présences « intermédiaires » en Maurienne et dans la région de l'Ainan. À ce propos, le passage de la Chronique de Novalaise est suggestif, lorsqu'il est question de la descente de Charlemagne en Italie :

Movens interea idem rex ingentem exercitum suum pervenitque in montem Geminum, sive ianuam regni Italie dici potest. In quo olim templum ad honorem cuiusdam cacodeo scilicet Iovis, ex quadris lapidibus, plumbo et ferro valde conexis, mire pulchritudinis, quondam constructum fuerat. In eo quoque montem due consurgunt fontes, una ex uno latere montis, alia ex alio [il s'agit de la Doire et de la Durance] sicque in convallibus suis descendentes et paulatim crescentes, magna efficiunt flumina ${ }^{65}$. 


\section{NOTES}

1. G. CANTino WATAGHin, «Between Franks and Lombards: Novalesa Abbey in the Early Middle Ages ", communication à Meeting the gentes - Crossing Boundaries: Columbanus and the Peoples of post-Roman Europe. International Round Table, Vienna, Institut für Mittelalterforschung, 22-23 novembre 2013 ; cf. aussi EAD., « "In loco nunccopante Novelicis" : La Novalesa dall'età romana alla fondazione di Abbone ", in Novalesa. Una storia tra fede e arte, Sant'Ambrogio di Torino, 2000, p. 11-31.

2. Pour une synthèse et une bibliographie mise à jour jusqu'à 2003, cf. G. SERGI, « Novalesa fra storia e storiografia ", in M. G. CERRI (dir.), Novalesa. Nuove luci dall'Abbazia, Milan, 2004, p. 21-33 et 169-171 ; cf. ensuite G. LUNARDI, I costruttori dell'Abbazia di Novalesa, Novalaise, 2003 ; ID., La congregazione sublacense O.S.B., Noci, 2003 ; G. BARRUOL et H. FALQUE-VERT, « Les biens du patrice Abbon en 739 ", in C. JOURDAIN-ANNEQUIN et M. LE BERRE (éd.), Atlas culturel des Alpes occidentales, 
Paris, 2004, n 126, p. 248-249; L. RIPART, La Novalaise, les Alpes et la frontière (VIII ${ }^{e}-\mathrm{XII}^{e}$ siècle), in F. ARNEODO et P. GUGLielmotTi (dir.), Attraverso le Alpi: S. Michele, Novalesa, S. Teofredo e altre reti monastiche, Bari, 2008, p. 95-114 ; ID., "L'abbaye de la Novalaise et son patrimoine provençal ", Bulletin de la Société d'études des Hautes-Alpes, 2015, p. 63-76; pour une synthèse des résultats des recherches archéologiques, cf. G. CANTINO WATAGHIN, «L'établissement et l'histoire de l'abbaye de Novalaise ", in M. LAUWERS (dir.), Monastères et espace social. Genèse et transformation d'un système de lieux dans l'Occident médiéval, Turnhout, 2014, p. 255-288.

3. Elles sont dues à l'aménagement dans les bâtiments du monastère d'un établissement hydrothérapeutique (G. VALERIO, La Novalesa antica abbazia, novella casa di salute, Turin, 1866), qui fit suite à la suppression au Piémont des ordres religieux en 1855 (A. BOGGE, «La soppressione dell'Abbazia di Novalesa nel 1855 e la vendita dei suoi beni", in Nuove scoperte alla Novalesa. Raccolta di studi presentati al convegno per il 1250esimo dell'atto di donazione di Abbone alla abbazia benedettina, Chieri, 1979, p. 111 sq.) et ensuite à l'installation d'un collège de Turin (G. PARATO, Novalesa, villa del Collegio Nazionale Umberto I di Torino, Turin, 1890 ; G. MONTICELLI, La valle di Susa e l'abbazia della Novalesa, dal 1884 villa del Convitto Nazionale di Torino: evocazione storica, Pinerolo, 1925).

4. Pour la reconstruction du XVIII siècle, cf. G. CARPIGNANO et E. RAGUSA, «Un esempio di intervento sui benefici vacanti: la chiesa e il palazzo abbaziale nel Settecento ", in La Novalesa. Ricerche - Fonti documentarie - Restauri, Suse, 1988, p. 241-295 ; le projet de restauration a fait suite à l'acquisition du monastère par l'administration provinciale de Turin en 1973 et au retour à Novalaise d'une communauté bénédictine; une synthèse des travaux dans L. PItTARello, «L'Abbazia della Novalesa negli ultimi cent'anni : dai primi riconoscimenti di interesse storico artistico ai lavori attuali e in corso ", in La Novalesa..., ibid., p. 397-432 et D. BIANCoLINI, « Novalesa : il lungo recupero della memoria ", in Abbazia dei SS. Pietro e Andrea di Novalesa. Dedicazione della Chiesa 15 ottobre 1995, Novalaise, 1995, p. 27-30; pour les détails des recherches archéologiques (fouilles et analyses du bâti), cf. G. CANTino WATAGHin, "L'établissement et l'histoire de l'abbaye... », op. cit., p. 258 sq.

5. G. TABACCO, "Dalla Novalesa a S. Michele della Chiusa", in Monasteri in Alta Italia dopo le invasioni saracene e magiare (secc. X-XII), Turin, 1966, p.479-526 (= ID., Spiritualità e potere nel medioevo. Dodici percorsi nei territori del potere e della fede, Naples, 1993, p. 11-74).

6. Ce «topos" est présent dans le Chronicon, selon lequel la fondation du monastère dans la «vallée de la Novalese... fort agréable et les habitants d'icele courtois et benins » est faite dans " un canton dans cette région du côté de midy, au pied d'une grande forest et sur un cousteau éloigné du passage des étrangers» (I, IV dans le fragm. I de G. Baldesano : G. C. ALEsSio (éd.), Cronaca di Novalesa, Turin, 1981, p. 10 et 12).

7. C. CARDUCCI, «Rilievi romani nell'abbazia della Novalesa », in Atti del V Congresso Nazionale di Archeologia Cristiana (Torino - Valle di Susa - Cuneo - Asti - Valle d'Aosta - Novara), 22-29 settembre 1979, Rome, 1982, p. 123-142, ici p. 125 ; mais contra déjà G. CANTINO WATAGHIN, « Il valico del Moncenisio in età romana : dati archeologici e ipotesi di lavoro ", in Le réseau routier en Savoie et en Piémont. Aspects historiques et contemporains [Bulletin de Centre d'études franco-italien, 8 (1981)], p. 27-33.

8. E. DE LEVIS, Raccolta di diverse antiche iscrizioni e medaglie epitalamiche ritrovate negli stati di S.S.R.M. il Re di Sardegna, e due dissertazioni sopra un antico turibolo, e campanello, Turin, 1781, p. 12, tav. IX. De l'inscription on lit Cassi[ia - - -]eami f(ilia) / Sev[er]a sibi et / Tito Qu[- - -]mio Quir(ina tribu) / [- - ]to[- - - ]/ - - e]st(- - -) (?) / - - - - - (?) / et A++[- - ]+[- - ]+[- - - ]++[- - ] f(ili-) : CIL V, 763, où pourtant la stèle est jugée médiévale ; E. CIMAROSTI, Le iscrizioni di età romana sul versante italiano delle "Alpes Cottiae", Barcelone, 2012 (Sylloge Epigraphica Barcinonensis, Annexos I), n 75, p. 246-248; la stèle (pour laquelle cf. C. FrAnzonI, Habitus habitusque militis,Rome, 1987, p. 98 sq.) se trouve aujourd'hui au Musée Archéologique de l'Abbaye (inv. $n^{\circ}$ 90081). Sur la même paroi étaient plaqués des fragments de reliefs du haut Moyen Âge, appartenant sans doute au mobilier 
liturgique de l'abbaye, qui n'entrent donc pas dans le problème dont il est question ici. Cette présentation s'insère sans doute dans le cadre des travaux dus au Convitto Nazionale Umberto I de Turin, qui, à la fin $\mathrm{du}_{\mathrm{XIx}} \mathrm{e}^{\mathrm{e}}$ siècle, devint propriétaire du monastère et $\mathrm{y}$ aménagea la résidence d'été des pensionnaires (cf. MonTicelli, La valle di Susa e l'abbazia..., op. cit.).

9. Musée Archéologique de l'abbaye, inv. n 90068 : [D(i)s] M(anibus / [- - ]iae / [- - -]lib(ertae) / [- - carissi]mae (?) / - - - - - (?) : E. CIMARostı, Le iscrizioni di età romana..., ibid., n 131, p. 329 sq.

10. Musée Archéologique de l'abbaye, inv. $n^{\circ}$ 90060-90063 ; 90065-90067; 90072 ; Torino, Museo di Antichità, deposito, inv. n 182-183 ; NA82 AVIII 2 ; cf. E. CIMAROSTI, Le iscrizioni di età romana..., ibid., $\mathrm{n}^{\circ}$ 168-171, 175-176, 180-181, 184, 191 et 195-196, p. 363-366, 369-370, 372-372, 375, 381 et $384 \mathrm{sq}$. : les datations proposées dans cette étude ne sont pas toujours convaincantes, notamment les nombreuses attributions à l'Antiquité tardive, qui ne paraissent pas entièrement justifiées ; on regrette d'ailleurs les nombreuses inexactitudes quant à la provenance des fragments.

11. G. LUNARDI, La congregazione sublacense..., op. cit et supra n. 3.

12. Musée Archéologique de l'abbaye, inv. n 90079 et 56949 ; pour les fragments de colonnes, cf. inv. $n^{\circ}$ 90162, 90163 et 90164 .

13. Musée Archéologique de l'abbaye, inv. $n^{\circ} 90094$.

14. Turin, Museo di Antichità, inv. 581.

15. M. TORELLI, « Il fregio d'armi nel Museo di Antichità di Torino. Ipotesi per un monumento di un senatore di epoca claudia ", in L. MERCANDo (dir.), Archeologia a Torino. Dall'età preromana all'Alto Medioevo, Turin, 2003, p. 151-169; à remarquer pourtant que, du point de vue stylistique, le relief de Novalaise ne correspond pas entièrement à ceux de Turin, contrairement à l'opinion de Carlo Carducci ("Rilievi romani nell'abbazia della Novalesa... », op. cit., p. 127 sq.).

16. Musée Archéologique de l'abbaye, inv. nº 90093.

17. La diffusion du thème est bien documentée dans E. POLITo, Fulgentibus armis. Introduzione allo studio dei fregi d'armi antichi, Rome, 1998, qui pourtant ne prend pas en compte le relief de Novalaise; cf. par contre A. BETORI, «Rilievi di soggetto storico dalla Val di Susa: alcune osservazioni », Quaderni della Soprintendenza Archeologica del Piemonte, 21 (2006), p. 153-166, ici p. 155 sqq.

18. M. LANGE, «Mensae ponderariae in Piemonte : studio dei reperti conservati nei monasteri della Novalesa e Villar San Costanzo », Quaderni della Soprintendenza Archeologica del Piemonte, 28 (2013), p. 79-88.

19. G. Cantino Wataghin, «L'abbazia dei Santi Pietro e Andrea di Novalesa: il contributo delle indagini archeologiche al recupero della sua memoria ", in M. G. CERRI (éd.), Novalesa. Nuove luci dall'Abbazia..., op. cit., p. 49. La couche a été enlevée sur une épaisseur de $2 \mathrm{~m}$ à l'aide d'une pelleteuse en 1974, au cours des premiers travaux d'aménagement des bâtiments du monastère, après l'arrivée de la communauté bénédictine, pour permettre l'accès des voitures à la partie orientale du cloître. Sa formation, artificielle, intègre l'abaissement du niveau du sol dans l'aménagement de caves et d'étables au lieu de l'ancien réfectoire qui occupait l'aile sud du cloître. Les fragments $\mathrm{du}$ bloc, cassé par la pelleteuse, ont été récupérés par dom Daniele Mazzucco, membre de la communauté bénédictine, auquel nous sommes redevables d'avoir suivi attentivement tous les nombreux travaux menés depuis 1973 en dehors d'un contrôle archéologique, en assurant ainsi la récupération de maints témoignages du passé de l'abbaye.

20. Musée Archéologique de l'abbaye, inv. n 90077 et 90078 : G. CANTINO WATAGHIN, « Il valico del Moncenisio... », op. cit., p. 28.

21. Musée Archéologique de l'abbaye, inv. nº 90099.

22. Musée Archéologique de l'abbaye, sans inv. : S. UGGÈ, Abbazia di Novalesa. Il Museo Archeologico, Abbaye de Novalèse, 2012 (Fragmenta Novaliciensia, 1), p. 48-49 et 51-54.

23. A. CRosetto, C. Donzelli et G. WATAghin CANTino, «Per una carta archeologica della Valle di Susa», Bollettino storico-bibliografico subalpino, 79 (1981), p.355-412, ici p. 408 sq.; G. CANTINO 
WATAGHIN, «Il valico del Moncenisio... », op. cit., p. 28 ; EAD., « "In loco nunccopante Novelicis”... », op. cit., p. 20. Nous ne prenons pas en compte le chaton d'anneau en pâte de verre, récupéré dans un niveau du haut Moyen Âge (S. UGGÈ, « Novalesa. Abbazia dei SS. Pietro e Andrea. Museo Archeologico ", Quaderni della Soprintendenza Archeologica del Piemonte, 25 (2010), p. 244-249, ici p. $242 \mathrm{sq}$.), dont le remploi vraisemblable dans le contexte du monastère ne garantit pas qu'il provienne du site même. Sur le remploi de gemmes romaines dans les productions somptuaires et leur présence dans les trésors ecclésiastiques et sur le contexte culturel et religieux de ces pratiques, cf. M. GREENHALGH, The Survival of Roman Antiquities in the Middle Ages, Londres, 1989, p. 229-232 ; J.-P. CAILLET, Les Trésors des sanctuaires, de l'Antiquité à l'époque romane, Paris, 1996 ; cf. aussi G. SENA CHIESA (dir.), Gemme. Dalla corte imperiale alla corte celeste, Milan, 2002, notamment G. SENA CHIESA, «Introduzione. Il prestigio dell'antico e il riuso glittico tra IV e X secolo », p. 1-16; les articles qui suivent dans le volume analysent les différents contextes de remploi et leurs modalités. Dans le cas spécifique de l'abbaye de Novalaise, les sources font mention du trésor accumulé par l'abbé Frodoinus, attesté en 773, qui «Cum quo etiam thesauro fecit crucem in eodem loco, auro argentoque, nec non gemmis preciosissimis oppido operatam ", [Chronicon, III, XVI, in C. CIPOLLA (éd.), Monumenta novaliciensia vetustiora, II, Rome, 1901 (Fonti per la Storia d'Italia, 32), p. 185 ; G. C. ALESSio (éd.), Cronaca di Novalesa, Turin, 1981, p. 160], à identifier sans doute avec celle mentionnée au XVII ${ }^{e}$ siècle par dom Rochex (J.-L. ROcHEX, La gloire de l'abbaye et vallée de la Novalaise située au bas du Montcinis du côté d'Italie, Chambéry, 1670, p. 66) : G. GENTILE, " Antichi arredi alla Novalesa ", in Nuove scoperte alla Novalesa..., op. cit., p. 81-105, ici p. 82 sq. ; sur les croix gemmées, cf. C. PelLegris, « Le croci gemmate dal V al XII secolo », in G. SENA CHIESA (dir.), Gemme. Dalla corte imperiale alla corte celeste..., ibid., p. 123-140.

24. Un autre sondage plus à l'ouest, au sud de l'aile méridionale du cloître, réalisé en 2006 par la Soprintendenza Archeologica del Piemonte, en vue de la mise en place d'une citerne, et resté inédit, a mis au jour des structures (cf. fig. 12), dont les niveaux d'occupation ont livré une quantité importante de vaisselle en pierre ollaire, actuellement en cours d'étude. Pour des informations préliminaires sur la présence de ce type de mobilier dans le contexte de l'abbaye, cf. G. GALLESIO et G. PANTò, « Proposte per una classificazione preliminare dei materiali dagli scavi dell'abbazia di Novalesa », in La Novalesa..., op. cit., p. 359-393, ici p. 360 sq.

25. C. CARDUCCI, «Rilievi romani nell'abbazia... », op. cit., p. 139 ; la provenance de la ville de Suse de la frise est soutenue aussi par L. MERCANDO, « La città, le mura, le porte », in L. MERCANDo (éd.), La porta del Paradiso. Un restauro a Susa, Turin, 1993, p. 61-136, ici p. 103 ; selon Betori, le fragment de frise et le bloc architectural «furono trasportati [à Novalaise] con ogni probabilità in età postantica » (A. BETORI, « Rilievi di soggetto storico... », op. cit., p. 154.

26. M. MARTINI, E. SIBILIA et G. SPINOLO, « Recent TL dating activity at Milan University: Datazione con il metodo della termoluminescenza di reperti provenienti dall'abbazia di Novalesa e dallo scavo del castello di Piadena ", in Y. LIRITZIS et T. HACKENS (éd.), Proceedings of the First Southern European Conference in Archaeometry, Strasbourg,1986 (PACT, revue du Groupe européen d'études pour les techniques physiques, chimiques et mathématiques appliquées à l'archéologie, 15), p. 127-131.

27. G. SCHMIEDT, "Le vicende dei transiti alpini dalla preistoria all'altomedioevo", in Le Alpi e l'Europa, t. 3 (Economia e transiti), Bari, 1975, p. 95-164 ; R. CHEVALLIER, Les voies romaines, Paris, 1997, p. 192-194; plus spécifiquement S. PADOVAN et E. TIRAULT, Chiomonte e Sollières. Pionieri delle Alpi dal Neolitico alle invasioni galliche / Pionniers des Alpes du Néolithique aux invasions gauloises, Turin, 2007 ; S. PADOVAN, «La Valle della Dora Riparia dal Neolitico all'età del Ferro. Linee di inquadramento cronologico e culturale ", in Lontane radici. Vaie, 60 secoli di storia, Borgone di Susa, 2010, p. 7-16 ; F. BARELLO, L. FERRERO et S. UGGÈ, « Evidenze archeologiche in Valle di Susa : acquisizioni, bilanci, prospettive di ricerca », Segusium, 50 (2013), p. 23-78, ici p. 24 sqq. 


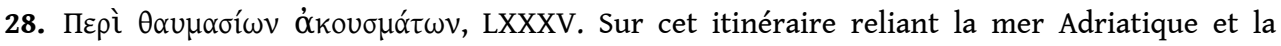
Méditerranée occidentale, présent dans la légende d'Héraklès et des Argonautes, et sur son identification avec la voie des Alpes Cottiennes, cf. R. DION, «La voie hérakléenne et l'itinéraire transalpin d'Hannibal», in M. RENARD (éd.), Hommages à Albert Grenier, Bruxelles, 1962, I, p. 527-543 ; L. BRACCESI, «Per una frequentazione dell'arco alpino occidentale (nota a Strab. 4, I, 3.178) », in Scritti storico-epigrafici in memoria di Marcello Zambelli, Macerata, 1978, p. 61-67 ; ID., «Eracle fra Celti e Liguri », in ID., Hellenikòs kolpos. Supplemento a "Grecità adriatica », Rome, 2001, p. 75-80, ici p. 78 sqq.; pour les données archéologiques, cf. M. VENTURINO GAMBARI, «Forme e dinamiche degli insediamenti umani nel Neolitico ", in L. MERCANDO et M. VENTURINO GAMBARI (dir.), Archeologia in Piemonte. La preistoria, Turin, 1998, p.101-121 ; EAD., "Società ed economia dal Neolitico all'età dei Metalli », ibid., p. 231-246 ; F. M. GAMBARI, « Elementi di organizzazione sociale ed economica delle comunità protostoriche piemontesi », ibid., p. 247-260 ; F. BARELLO, L. FERRERO et S. UGGÈ, « Evidenze archeologiche... », op. cit.

29. K. MILLER, Itineraria romana, Stuttgart,1916, p. 96 ; G. RADKE, « Römische Strassen in der Gallia Cisalpina und der Narbonensis ", Klio, 42 (1964), p. 299-317 ; ID., Viae publicae Romanae, Bologne, 1981 (= Stuttgart, 1971 et in RE, XIII, 1973, c. 1417-1686) ; R. CHEVALLIER, Les voies romaines..., op. cit., p. $53 \mathrm{sqq}$.

30. La thèse remonte au XIX ${ }^{\mathrm{e}}$ siècle $:$ L. CIBRARIO, « La route du Piémont en Maurienne ", Mémoire de l'Académie de Savoie, 4 (1830), p. 194-211; H. NISSEN, Italische Landeskunde, t. 1, Berlin,1883, p. 158 ; elle a été ensuite reprise, entre autres, par W.W. HYDE, Roman Alpine Routes, Philadelphie, 1935, p. 55 sq. ; P. BAROCELLI, La via romana transalpina degli alti valichi dell'Autaret e di Arnas. Note di escursioni archeologiche nelle valli di Lanzo, Turin,1968, p. 6 sqq.; C. CARDUCCI, "Études sur l'identification des cols alpins entre Piémont et Gaule transalpine dans le cadre des découvertes archéologiques ", in Actes du colloque international sur les cols des Alpes, Orléans, 1971, p. 45-52 ; jusqu'aux plus récents R. CHEVALLIER, Les voies romaines..., ibid.; R. SCUDERI, "Confine amministrativo e confine doganale nelle Alpi occidentali durante l'alto impero ", in S. G IORCELLI BERSANI (éd.), Gli antichi e la montagna - Les anciens et la montagne, Turin, 2001, p. 167-183, ici p. 180, n. 72 ; contra très brièvement et sans suite réelle D. GRIBAUDI, Il Piemonte nell'antichità classica, Turin, 1928, p. 126 sq.; G. CORRADI, Le strade romane dell'Italia nordoccidentale, Turin, 1939, n. 110 à la p. 56 de l'édition de 1968.

31. Comme en témoignent les nombreux postes de la quadragesima Galliarum, qui ne sont pas enregistrés dans les itinéraires : cf. S. J. DE LAET, Portorium. Étude sur l'organisation douanière chez les Romains, surtout à l'époque du Haut-Empire, Bruges, 1949, toujours fondamental ; plus récemment G. MENNELlA, «La "Quadragesima Galliarum" nelle “Alpes Maritimae” ", Mélanges de l'École française de Rome, Antiquité, 104 (1992), p. 209-232 ; R. sCUDERI, «Confine amministrativo... », op. cit., p. 281 $s q q$.

32. J. PRIEUR, «Un habitat au pied du col du Mont-Cenis : Lanslevillard, du néolithique à la fin de l'époque romaine ", in Atti del Centro Studi e Documentazione sull'Italia Romana, t. 7 (1975-1976), p. 521-534; G. CANTINO WATAGHIN, « Il valico del Moncenisio in età romana... », op. cit.

33. G. MENNELla, «Un'iscrizione rupestre dal Moncenisio », in Saxa Scripta. Actas do III Simpósio Ibero-itálico de Epigrafia rupestre, Viseu, 2001, p. 119-136 ; ID., «La Roccia degli Stambecchi. Scene di caccia e iscrizioni votive di età romana fra Moncenisio e Monginevro », in A. ARCÀ (éd.), La Spada sulla Roccia. Danze e duelli tra arte rupestre e tradizioni popolari della Valsusa, Valcenischia e delle valli del Moncenisio, Turin, 2009, p. 27-32; A. ARCÀ, F. M. GAMBARI et G. MENNELLA, «La roccia degli stambecchi : un'iscrizione latina reinterpreta incisioni dell'età del Ferro ", in Secondo convegno internazionale di archeologia rupestre: l'Europa - le Alpi - la Valcamonica, Milan, 2001, p. 83-90. Les trois inscriptions donnent une interprétation érotique aux images de chasse, cf. F. M. GAMBARI, «La caccia signorile in quota : una pratica rituale dell'età del Ferro ? ", in A. ARCÀ (éd.), La Spada 
sulla Roccia..., op. cit., p. 19-26. Le contrôle de la contrebande le long des pistes qui desservaient les multiples passages des Alpes, entre Gaule et Italie, évitant les routes majeures où les stationes de la Quadragesima Galliarum étaient situées, était un problème bien réel pour l'administration romaine : cf. infra, n. 45.

34. Musée Archéologique de l'abbaye, inv. $n^{\circ} 90165$; le fragment était en œuvre dans un mur en pierres sèches à l'est du cloître; un fragment d'un autre milliaire était remployé dans les maçonneries de l'église paroissiale du village de Novalaise : cf. G. CANTINO WATAGHIN, «In loco nunccopante Novelicis...", op. cit., p. 23; de nombreuses pièces de comparaison dans le recensement de E. BANZI, I miliari come fonte topografica e storica. L'esempio della XI Regio (Transpadana) e delle Alpes Cottiae, Rome, 1999, que nous n'avions pas pu consulter alors ; il est mentionné dans E. CIMARostI, "Verso il confine : un aggiornamento ai miliarii taliani delle Alpes Cottiae », in P. LEVEAU et B. RÉMY (dir.), La ville des Alpes occidentales à l'époque romaine, Grenoble, 2008, p. 207-223, ici p. 207, n. 11.

35. J. PRIEUR, «Les voies transalpines entre le petit Saint-Bernard et le Mont-Genèvre à l'époque romaine. État des questions ", in Le Mont-Cenis et sa région, Chambéry, s.d. [1979], p. 373-386 ; d'un « système de cols » (« sistema di valichi ») parle à juste titre Sergi (G. SERGI, Potere e territorio lungo la strada di Francia. Da Chambéry a Torino fra X e XIII secolo, Naples, 1981, p. 35 sq.), dans une perspective qui montre l'inconsistance de la théorie proposée par de Lavis-Trafford et largement adoptée par les chercheurs, d'un passage qui se serait fait seulement à travers le Petit-MontCenis dans l'Antiquité et jusqu'au Moyen Âge, quand la route du Grand-Mont-Cenis aurait été ouverte (M. DE LAVIS TRAFFORD, "L'identification topographique du col alpin franchi par Hannibal », Travaux de la Société d'histoire et d'archéologie de Maurienne, 13 (1956), p. 109-152; ID., «Études sur les voies transalpines de la région du Mont Cenis, depuis l'antiquité classique jusqu'au début du XIII ${ }^{\mathrm{e}}$ siècle », Bulletin philologique et historique, 1 (1960), p. 61-91.

36. C. LETTA, «La dinastia dei Cozii e la romanizzazione delle Alpi », Athenaeum, 54 (1976), p. 36-76; ID., «La dynastie royale des Alpes Cottiennes », in Le Mont-Cenis et sa région..., op. cit., p. 303-323; ID., «Postilla sulle iscrizioni della dinastia cozia», in Susa. Bimillenario dell'arco = Segusium, 31 (1994), p. 115-127.

37. L. MERCANDO, « La città, le mura... », op. cit. Dans un article qui vient d'être publié, Anna Maria Riccomini a supposé l'existence à Suse d'un complexe statuaire d'époque claudienne, résultat d'un programme de propagande dynastique, auquel, d'une part, appartiendraient les deux torses cuirassés retrouvés au début du XIX ${ }^{\mathrm{e}}$ siècle et auquel, d'autre part, serait reliée la tête conservée à New York (Metropolitan Museum), traditionnellement identifié avec Agrippa, mais qui pourrait plutôt représenter Germanicus, le lien de Claude avec la "gens Iulia » ou plutôt Marc Antoine, aïeul de l'empereur [A. M. RiccominI, «Sul ritratto in bronzo da Susa al Metropolitan Museum (New York). Una possibile identificazione : Marco Antonio ? », Segusium, 52 (2015), p. 303-316].

38. SUETONE, Nero, 18 ; J. PRIEUR, La province romaine des Alpes Cottiennes, Villeurbanne, 1968 ; C. LETTA, «La dinastia dei Cozii e la romanizzazione... », op. cit. ; ID., «La dynastie royale des Alpes... ", op. cit.; G. CRESCI MARRONE, "Segusio e il processo di integrazione nella romanità », Segusium, 31 (1994), p. 185-196.

39. Segm. II, 3 : L. Bosio, Tabula Peutingeriana : una descrizione pittorica del mondo antico, Rimini, 1983, fig. 47 et p. 157, n. 294 ; pour une analyse nouvelle et stimulante de la Table, cf. R.J. A. TALBERT, Rome's World : The Peutinger Map Reconsidered, Cambridge, 2010.

40. Amm. Marc. XV, 2: « quas [Alpes Cottiae] rex Cottius, perdomitis Gallis solus in angustiis latens inviaque locorum asperitate confisus, lenito tandem timore in amicitiam Octaviani receptus principis, molibus magnis exstruxit ad vicem memorabilis muneris, compendiarias et viantibus oportunas, medias inter alias Alpes vetustas super quibus comperta paulo postea referemus... et licet haec, quam diximus viam, media sit et conpendiaria magisque celebris, tamen etiam aliae multo antea temporibus sunt constructae diversis. » 
41. A. CRosetto, « La chiesa "Sancti Maximi ad quintum di Collegno ", in G. P. BRogiolo (éd.), Chiese e insediamenti nelle campagne tra V e VI secolo, $9^{\circ}$ seminario sul tardoantico e l'alto medioevo, Mantova, 2003, p. 119-130; ID., «La chiesa di S. Massino "ad Quintum": fasi paleocristiane e altomedievali », in L. PEJRANI BARICCO (dir.), Presenze Longobarde. Collegno nell'altomedioevo, Turin, 2004, p. 249-270 ; sur le problème du développement des stations routières dans l'Antiquité tardive, cf. G. CANTINO WATAGHIN, V. FIOCCHI NICOLAI et G. VOLPE, « Aspetti della cristianizzazione degli agglomerati secondari », in R. M. BONACASA CARRA et E. VITALE (éd.), La cristianizzazione in Italia fra tardoantico e altomedioevo, Palerme, 2007, p. 85-134, p. 94 sqq., p. 96 pour Collegno.

42. M. SEGARD, Les Alpes occidentales romaines, développement urbain et exploitation des ressources des régions de montagne (Gaule Narbonnaise, Italie, provinces alpines), Aix-en-Provence, 2009, p. 89-91 et $98 \mathrm{sqq}$.

43. C. CIPOLla (éd.), Monumenta novaliciensia vetustiora..., op. cit., p. 104 sqq. ; D. OLIVIERI, Dizionario di toponomastica piemontese, Brescia, 1965, p. 238.

44. Devant l'absence d'études spécifiques sur le terroir du Val Cenischia, on se reportera à M. SEGARD, Les Alpes occidentales romaines..., op. cit.; l'exploitation des ressources minières (cuivre et fer) est bien attestée au Moyen Âge, cf. G. DI GANGI, L'attività mineraria e metallurgica nelle Alpi Occidentali Italiane nel Medioevo: Piemonte e Valle d'Aosta, fonti scritte e materiali, Oxford, 2001, notamment p. 153-167 et p. 1 sqq. pour le potentiel minier; pour l'Antiquité et le haut Moyen Âge, on attend encore une enquête qui vérifie les suggestions des érudits du XIX ${ }^{e}$ siècle, résumées dans ibid., tav. 5.

45. CIL, XII 80 ; cette inscription a été étudiée surtout du point de vue de ses implications pour l'histoire administrative de ce secteur des Alpes: A. ROTH CONGÈs, "L'inscription des Escoyères dans le Queyras, la date de l'octroi du droit latin aux Alpes Cottiennes, et la question du statut de Dinia ", Rivista di Studi Liguri, 59-60 (1993-1994), p.73-101, avec bibliographie à la n. 2; C. Letta, « Ancora sulle civitates di Cozio e sulla prefettura di Albano », in S. GIORCELLI BERSANI (éd.), Gli antichi e la montagna..., op. cit., et encore B. RÉMY et F. KAYSER, «Inscriptions latines des Alpes : Alpes Cottiennes (versant français) ", in E. CIMARosTI, Le iscrizioni di età romana..., op. cit., p. 355-611, ici p. 598-603 ; M. M. FALCHI, "La présence humaine aux Escoyères dans l'Antiquité. Quelques observations sur l'inscription de la chapelle de Sainte Marie-Madeleine », Bulletin de la Société d'études des Hautes-Alpes (2015), p. 29-46, qui restitue une stèle plutôt qu'un monument funéraire et relie son érection à l'activité du commanditaire dans le service des douanes.

46. Les deux fragments de l'inscription sont réemployés comme linteaux de porte dans la chapelle Sainte-Madeleine du hameau, situé dans la commune d'Arvieux; d'autres matériaux ayant appartenu au mausolée, notamment des pierres de taille, sont également réutilisés dans la chapelle: G. BARRUOL, Les peuples préromains du sud-est de la Gaule, étude de géographie historique, Paris, 1969, p. 44-48 et 175-179; I. GANET (dir.), Carte archéologique de la Gaule, t. 5 (Hautes-Alpes), Paris, 1995, « 007. Arvieux », p. 52 sq.

47. G. BÉRARD (dir.), Carte archéologique de la Gaule, t. 4 (Les Alpes-de-Haute-Provence), Paris, 1997, «090. Le Fugeret », p. 207-209; M. SEGARD, Les Alpes occidentales romaines..., op. cit., p. 237.

48. Cf. supra, n. 26.

49. Cf. supra, n. 24.

50. S. UGGÈ, "Pettine e placca in osso (astuccio di pettine ?) ", in F. CRIVELlo et C. SEGRE MONTEL (dir.), Carlo Magno e le Alpi. Viaggio al centro del Medioevo, Milan, 2006, p. 88-89.

51. G. SARONI, « Cofanetto reliquiario in osso ", in Novalesa. Una storia..., op. cit., p. 107-109; EAD., «Reliquiari della Novalesa e della cattedrale di Vercelli. Cofanetto reliquiario in osso », in C. BERTELLI et G. P. BRogiolo (dir.), Il futuro dei Longobardi. L'Italia e la costruzione dell'Europa di Carlo Magno, Milan, 2000, p. 222 (scheda $n^{\circ} 254$ ). La chasse elle-même est datée de la seconde moitié du $\mathrm{XIII}^{\mathrm{e}}$ siècle ; elle a été ouverte en 1998, en vue de restaurations ; outre le reliquaire, dont il est question, elle renfermait les reliques de saint Eldrade, saint Arnulfe et autres saints et trois petits 
reliquaires en bois peint du XI-XII ${ }^{\mathrm{e}}$ siècle : C. BERTOLOTTO, « La cassa di Sant'Eldrado e i reliquiari ritrovati », in Novalesa. Una storia..., ibid., p. 97-106.

52. Les trésors des Églises de France, catalogue d'exposition, musée des Arts décoratifs, Paris, 1965, p. 226 , fiche $n^{\circ} 412$.

53. Le coffret renfermait des reliques arrivées à l'abbaye à différentes époques: les saints Côme et Damien (selon la Chronique, III, 15, données par Charlemagne : G. C. ALEssio, Cronaca, p. 160-161), André, Nicolas, Théobald, Vincent (C. BERTolotto, «La cassa di Sant'Eldrado e i reliquiari ritrovati », op. cit., p. 97-106, ici p. 98, n. 3). Le problème de la date de sa présence dans la ville se pose aussi pour l'exemplaire très proche du Trésor de la Cathédrale de Suse: S. UGGÈ, « Reliquiario in osso », in F. CRIVello et C. Segre montel (dir.), Carlo Magno e le Alpi..., op. cit., p. 92-93. 54. G. ARDIZIO et G. CANTINOWATAGHIN, «Ulteriori note su alcuni frammenti di sarcofagi paleocristiani dall'abbazia dei SS. Pietro e Andrea di Novalesa », Quaderni della Soprintendenza Archeologica del Piemonte, 26 (2011), p. 65-76.

55. Trois fragments d'inscriptions, retrouvés à différents moments, sont conservés : deux à la Novalaise (Musée Archéologique, inv. $n^{\circ}$ 56953-56954), un à Turin (Palazzo Madama Museo Civico di Arte Antica, inv. $n^{\circ} 389,5$ et $\left.306 a\right)$.

56. La datation au haut Moyen Âge ressort de l'analyse détaillée menée récemment par Chiara Lambert (C. LAMBERT, « La scrittura epigrafica nei monasteri alpini : alcune linee di ricerca », in E. DESTEFANIS et C. LAMBERT (éd.), Per diversa temporum spatia. Scritti in onore di Gisella Cantino Wataghin, Vercelli, 2011, p.121-142, ici p. 131 sqq.); pour une chronologie se rapportant à l'Antiquité tardive, cf. C. CARDUCCI, «Rilievi romani...», op. cit., p.137; M. AIMONE, "Epitaffi tardoantichi e intrecci altomedievali. Osservazioni su tre frammenti iscritti e scolpiti provenienti dall'abbazia della Novalesa ", Bollettino storico-bibliografico subalpino, 108 (2010), p. 115-142 (où pourtant la cohérence des trois fragments n'est pas reconnue); C. LETTA, « Minima epigraphica dalle Alpes Cottiae. In memoria di Jacques Debergh: un carmen epigraphicum cristiano dalla Novalesa », Studi Classici e Orientali, 57 (2011), p. 315-325 et Segusium, 52 (2013), p. 11-22 ; dans un souci d'exhaustivité, rappelons aussi qu'une datation à la Renaissance a été proposée par L. MALLÈ, Le sculture del Museo di Arte Antica, Turin, 1965, p. 68S, suivi par S. CASARTELl NOVELLI, La diocesi di Torino. Corpus della scultura altomedievale, t. 6, Spolète, 1974, p. 174.

57. G. CANTino WATAGHIN, «L'abbazia dei SS. Pietro e Andrea di Novalesa: gli edifici monastici nell'altomedioevo ", in H. R. SENNHAUSER (dir.), Wohn- und Wirtschaftsbauten frühmittelalterlicher Kloster. Internationales Symposium, 26.9.-1.10.1995 in Zurzach und Müstair, in Zusammenhang mit den Untersuchungen im Kloster St. Johann zu Müstair, Zürich, 1996, p. 17-26; EAD., «L'établissement et l'histoire... ", op. cit., p. 263 sq. ; L. PEJRANI BARIcCo, s. UGGÈ et G. CANTINO WATAGHIN, « Novalesa. Scavi nell'Abbazia dei SS. Pietro e Andrea ", Quaderni della Soprintendenza Archeologica del Piemonte, 25 (2010), Notiziario, p. 236-243.

58. G. CANTINO WATAGHIN, « Architecture et décor peint de la Novalaise du carolingien au roman », in E. VERGNOLLE et S. BULLY (dir.), Le "premier art roman" cent ans après. La construction entre Saône et Pô autour de l'an Mil, Besançon, 2012, p. 239-259 ;EAD., « L'établissement et l'histoire... », ibid.

59. F. L. GANSHOF, «Quelques aspects principaux de la vie économique dans la monarchie franque au VII ${ }^{\mathrm{e}}$ siècle ", in Caratteri del secolo VII in Occidente, Spolète, 1958 (Settimana di studio del CISAM, V), p. 73-101.

60. J.-M. CARRIÉ, « Nommer les structures rurales entre fin de l'Antiquité et haut Moyen Âge : le répertoire lexical gréco-latin et ses avatars modernes, $1^{\mathrm{re}}$ partie », Antiquité Tardive, 20 (2012), p. 25-46, ici p. 30 sqq.

61. Je remercie Alain Dubreucq pour ses suggestions sur ce point, faites à l'occasion du séminaire de Vienne.

62. P. J. GEARY, Aristocracy in Provence. The Rhône Basin at the Dawn of the Carolingian Age, Stuttgart, 1985 ; G. BARRUOL et H. FALQUE-VERT, « Les biens du patrice Abbon en 739... », op. cit. ; E. DESTEFANIS et 
S. UGGÈ, « Possedimenti di Novalesa secondo il testamento di Abbone 739 », in F. CRIVELLo et C. SEGRE MONTEL (dir.), Carlo Magno e le Alpi..., op. cit., p. 48-49.

63. «(...) una cum consensu pontificum vel clerum nostrorum Mauriennate vel Segucine civitate in quibus nos Deus rectorem esse instituit » : C. CIPOLLA, Monumenta Novaliciensia Vetustiora, t. 1, Rome, 1898, doc. I, 726, gennaio 30, p. 7-13, ici p. 7.

64. P.J. GEARY, Aristocracy in Provence..., op. cit., p. 81 ; le testament fait mention de propriétés paternelles dans la ville de Suse : «(...) in ualle sigusina, tam infra muros ipsius civitatis quam et in ipso pago ex alode parentum meorum uel undecumque michi iustissime ibidem ex legibus obuenit, hoc est quidquid in ipsa ualle novaliciis (...) » (GEARY, Aristocracy in Provence..., op. cit., p. 40 sqq.).

65. Chronicon, III, 7 : G. C. ALESSIO, Cronaca..., op. cit., p. 145.

66. Pour la Chronique, cf. P. J. GEARY, La mémoire et l'oubli à la fin du premier millénaire, Paris, 1996 (=Phantoms of Remembrance : Memory and Oblivion at the End of the First Millennium, Princeton, 1996); le «templum (...) mirae pulchritudinis» évoqué par le chroniqueur a sans doute existé au col du Montgenèvre, des éléments d'un bâtiment important ont été retrouvés à l'occasion de la fouille du chœur de l'église paroissiale, restée inédite.

67. Pour une analyse précise de la figure d'Abbon et du contexte dans lequel il agit, cf. toujours P. J. GEARY, Aristocracy in Provence..., op. cit., p. 101 sqq.

68. En dernier lieu,L. RIPART, «L'abbaye de la Novalaise... », op. cit., p. 64 sqq.

69. Cf. W. KURZE, Monasteri e nobiltà nel Senese e nella Toscana medievale. Studi diplomatici, archeologici, genealogici, giuridici e sociali, Sienne, 1989, p. 303-312; G. SERGI, I confini del potere. Marche e signorie fra due regni medievali, Turin, 1995, p. 11.

70. C. SERENO, «Monasteri aristocratici subalpini : fondazioni funzionariali e signorili, modelli di protezione e di sfruttamento (secoli X-XII) (parte prima) », Bollettino storico-bibliografico subalpino, 96 (1998), p. 397-448, ici p. 421 [en ligne : www-rm.unina.it] ; G. SERGI, L'aristocrazia della preghiera. Politica e scelte religiose nel medioevo italiano, Rome, 1994, p. 55-72.

71. C. CIPOlla (éd.), Monumenta novaliciensia vetustiora,t. 1, Rome, 1898 (Fonti per la Storia d'Italia, 31), doc. I, 726 gennaio 30, p. 7-13, ici p. 9 ; à la famille d'Abbon appartiennent sans doute le premier abbé, un autre Abbon, et l'évêque Walchunus, qui signe l'acte de fondation du monastère et participe à son établissement matériel et auquel l'exécution du testament est confiée : P. J. GEARY, Aristocracy in Provence..., op. cit.; G. SERGI, « Novalesa fra storia e storiografia... », op. cit., p. 21 sq.

72. La définition est d'A. Perret («Les origines de l'expansion monastique en Savoie ", Mémoires de l'Académie des sciences, belles-lettres et arts de Savoie, 1 (1953), p. 29-69, ici p. 43), reprise par G. Sergi (« Novalesa fra storia e storiografia... », ibid., p. 28 sq.).

\section{AUTEUR}

\section{GISELLA CANTINO WATAGHIN}

Professeur honoraire, université du Piémont oriental 